\title{
Insights into the correlation between Physiological changes in and seed development of tartary buckwheat (Fagopyrum tataricum Gaertn.)
}

Moyang Liư ${ }^{\dagger}$ Zhaotang $\mathrm{Ma}^{\dagger}$, Tianrun Zheng, Wenjun Sun, Yanjun Zhang, Weiqiong Jin, Junyi Zhan, Yuntao Cai, Yujia Tang, Qi Wu, Zizhong Tang, Tongliang Bu, Chenglei Li and Hui Chen* (D)

\begin{abstract}
Background: Tartary buckwheat (Fagopyrum tataricum Gaertn.) is a widely cultivated medicinal and edible crop with excellent economic and nutritional value. The development of tartary buckwheat seeds is a very complex process involving many expression-dependent physiological changes and regulation of a large number of genes and phytohormones. In recent years, the gene regulatory network governing the physiological changes occurring during seed development have received little attention.

Results: Here, we characterized the seed development of tartary buckwheat using light and electron microscopy and measured phytohormone and nutrient accumulation by using high performance liquid chromatography (HPLC) and by profiling the expression of key genes using RNA sequencing with the support of the tartary buckwheat genome. We first divided the development of tartary buckwheat seed into five stages that include complex changes in development, morphology, physiology and phytohormone levels. At the same time, the contents of phytohormones (gibberellin, indole-3-acetic acid, abscisic acid, and zeatin) and nutrients (rutin, starch, total proteins and soluble sugars) at five stages were determined, and their accumulation patterns in the development of tartary buckwheat seeds were analyzed. Second, gene expression patterns of tartary buckwheat samples were compared during three seed developmental stages (13, 19, and 25 days postanthesis, DPA), and 9765 differentially expressed genes (DEGs) were identified. We analyzed the overlapping DEGs in different sample combinations and measured 665 DEGs in the three samples. Furthermore, expression patterns of DEGs related to phytohormones, flavonoids, starch, and storage proteins were analyzed. Third, we noted the correlation between the trait (physiological changes, nutrient changes) and metabolites during seed development, and discussed the key genes that might be involved in the synthesis and degradation of each of them.
\end{abstract}

Conclusion: We provided abundant genomic resources for tartary buckwheat and Polygonaceae communities and revealed novel molecular insights into the correlations between the physiological changes and seed development of tartary buckwheat.

Keywords: nutrition, phytohormones, RNA sequencing (RNA-seq), seed development, tartary buckwheat, transcriptome

\footnotetext{
* Correspondence: chenhui@sicau.edu.cn

${ }^{+}$Moyang Liu and Zhaotang Ma contributed equally to this work.

College of Life Science, Sichuan Agricultural University, Ya'an, China
}

(c) The Author(s). 2018 Open Access This article is distributed under the terms of the Creative Commons Attribution 4.0 International License (http://creativecommons.org/licenses/by/4.0/), which permits unrestricted use, distribution, and reproduction in any medium, provided you give appropriate credit to the original author(s) and the source, provide a link to the Creative Commons license, and indicate if changes were made. The Creative Commons Public Domain Dedication waiver (http://creativecommons.org/publicdomain/zero/1.0/) applies to the data made available in this article, unless otherwise stated. 


\section{Background}

Tartary buckwheat (Fagopyrum tataricum Gaertn.) comprises twenty different species of extensively cultivated medicinal and edible crop species with excellent economic and nutritional values [1, 2]. Tartary buckwheat was domesticated in East Asia and is cultivated in Europe and North America [3]. Today, it is common in the Himalayan region and Southwest China, such as Sichuan Province. In 2016, global production was 2.3 million tons, with Russia and China accounting for 50\% and $17 \%$ of the world's total output, respectively (adopted from UN Food and Agriculture Organization, Corporate Statistical Database: http://www.fao.org/). Tartary buckwheat seeds are a rich source of four B vitamins, dietary fiber, protein and a variety of minerals, in which niacin, magnesium, manganese, phosphorus content is particularly high (adopted from USDA Food Composition Databases: https://ndb.nal.usda.gov/). Importantly, tartary buckwheat seeds are known for their high rutin content, which has been proven to be effective in preventing liver injury and especially inflammatory liver injury [4].

The development of most seeds can be divided into three stages: tissue differentiation, cell enlargement and mature dehydration. At the stage of tissue differentiation, a single-cell zygote forms a young embryo composed of cotyledons by cell division and differentiation $[5,6]$. Seed development in higher plants is a very complex process involving many expression-dependent physiological changes and regulation of a large number of genes and phytohormones, and the development of tartary buckwheat seeds is no exception.

Although the regulatory networks of monocotyledonous plants (i.e. maize) seed development [7] and various nutritional and pharmacological effects of tartary buckwheat have been well studied [8-11], there are few reports on the gene regulatory network governing the physiological changes occurring during seed development of tartary buckwheat of dicotyledonous plants supported by the tartary buckwheat genome. Here, we characterized the seed development of tartary buckwheat using light and electron microscopy, quantification of phytohormone and nutrient accumulations using HPLC, and by profiling the expression of key genes through transcriptome profiling. By analyzing the correlation between phytohormone changes and seed development, we investigated embryo cell enlargement and primary dormancy of tartary buckwheat seeds. By analyzing the correlation between nutrient changes and seed development, we determined the gene regulatory network controlling the accumulation of rutin, starch, storage protein and soluble sugars during the development of tartary buckwheat seeds and analyzed the key genes. We provided abundant genomic resources for tartary buckwheat and Polygonaceae communities and revealed novel molecular insights into the correlations between the physiological changes and seed development of tartary buckwheat.

\section{Results \\ In-depth description of the development of tartary buckwheat seed}

The development of tartary buckwheat seed from anthesis to maturation was divided into five stages that include complex changes in development, morphology, physiology and phytohormone levels (Fig. 1a, b). The young fruit stage was 0-8 DPA, and the globular embryo gradually formed. At this time, the ovary cavity of the fruit was larger, and the seed was smaller, presenting light green coloration with deep grooves. During 8-14 DPA in the green fruit stage, the heart-shaped embryo formed in steps, the proportion of seeds in the fruit increased, the color of the fruit deepened, and the grooves became shallower. It is worth mentioning that the longitudinal diameter of the fruit reached the maximum at this time. At the discoloration stage (14-22 DPA), the fruit size reached the largest, the green color was the deepest, and the grooves were not obvious; the torpedo-shaped embryo also formed at this time. At the initial maturity stage (22-26 DPA), fruit weight reached the maximum, and the fruit began to show signs of water loss, turn yellow in color, became smaller in size, and developed deeper grooves. The mature stage was 26-30 DPA, during which time the color of the seed turned dark yellow, the seed filled up the entire fruit, and the cotyledon changed from transparent to white, showing the maturity of the embryo. The shape of the developing embryo in each developing seed is a more reliable time marker [12]. Based on the number of days postanthesis, the young fruit stage to maturity stage described here correspond to the globular embryo to the mature embryo in tartary buckwheat seeds.

\section{Phytohormone accumulation at different stages of seed development}

In buckwheat seeds, the content of gibberellin $\left(\mathrm{GA}_{3}\right)$ was at a high level during the young fruit stage and peaked at 13 DPA with a value of $109.76 \mu \mathrm{g} \mathrm{g}^{-1}$ fresh weight $(\mathrm{FW})$. After a sharp reduction until the discoloration stage, the content of $\mathrm{GA}_{3}$ decreased to the lowest value of $25.28 \mu \mathrm{g} \mathrm{g}^{-1} \mathrm{FW}$. The second increase occurred before the initial maturity stage and reached a maximum of $117.34 \mu \mathrm{g} \mathrm{g}^{-1} \mathrm{FW}$ at 19 DPA. Throughout the development process, the $\mathrm{GA}_{3}$ content appeared as a fluctuation curve (Fig. 2a). The level of indole-3-acetic acid (IAA) also appeared as a fluctuation curve. The IAA content was high at young fruit stage and decreased sharply at green fruit stage. The second increase occurred before the discoloration stage and reached a value of $20.17 \mu \mathrm{g} \mathrm{g}^{-1}$ FW at 19 DPA, after which it decreased 

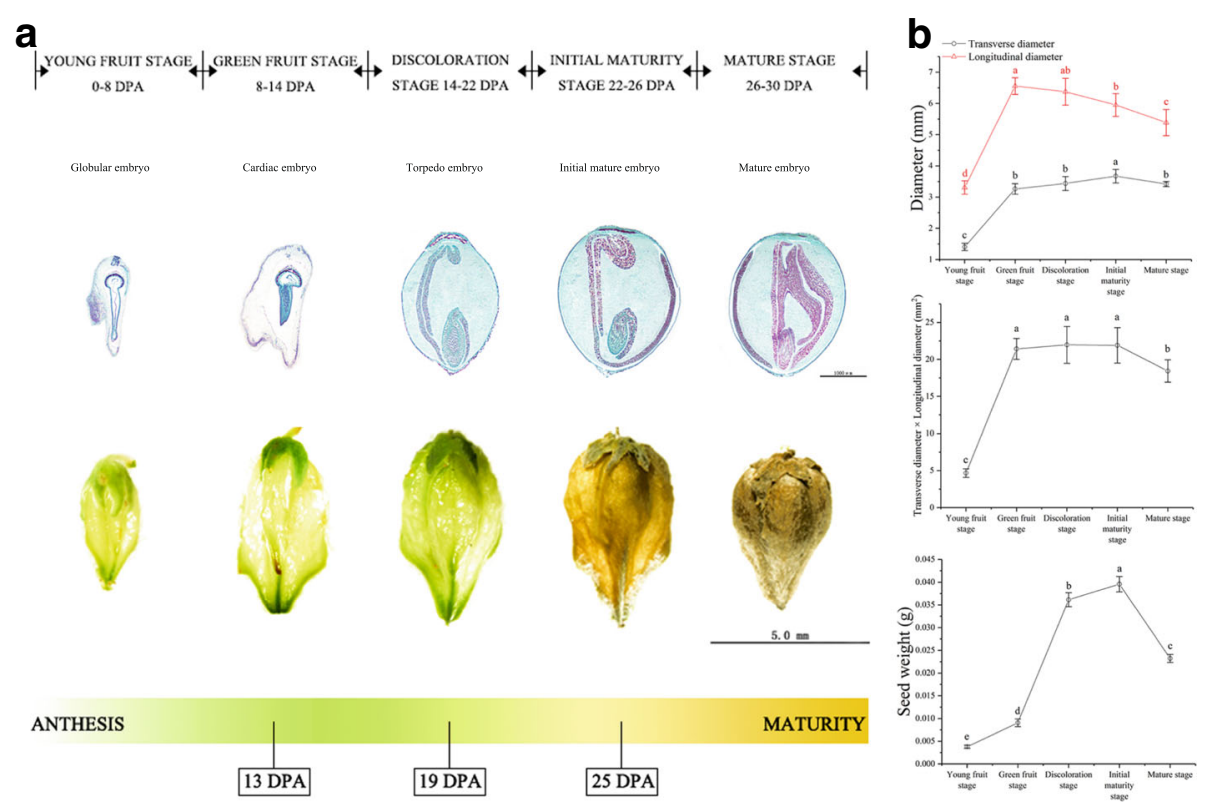

Fig. 1 Observation and measurement of the development of tartary buckwheat seeds. a Five stages of tartary buckwheat seeds development. Top row shows each longitudinal sections of tartary buckwheat seeds at different developmental stages. Second row shows individual seeds at different developmental stages. Third row shows schematic representation of developmental stages studied during tartary buckwheat seed ontogeny. 13 DPA: Tartary buckwheat seed at green fruit stage for transcriptome experiment; 19 DPA: Tartary buckwheat seed at discoloration stage for transcriptome experiment; 25 DPA: Tartary buckwheat seed at initial maturity stage for transcriptome experiment. DPA, days-post-anthesis. $\mathbf{b}$ The seed size and weight at different developmental stages. Error bars were obtained from five measurements. Small letter(s) above the bars indicate significant differences $(a=0.05, L S D)$ among treatments

again from this time to the initial maturity stage, with the lowest value of $5.56 \mu \mathrm{g} \mathrm{g}^{-1} \mathrm{FW}$. After a sharp reduction, it started to increase again to a content of $6.05 \mu \mathrm{g}$ $\mathrm{g}^{-1}$ FW (Fig. 2b). The content of zeatin (ZT) was at a high level during the young fruit stage and reached a peak value of $9.08 \mu \mathrm{g} \mathrm{g}^{-1} \mathrm{FW}$, after which it decreased sharply before the initial maturity stage. The content of ZT decreased to the lowest value of $0.41 \mu \mathrm{g} \mathrm{g}{ }^{-1} \mathrm{FW}$, but after a sharp reduction before the maturity stage, it started to rise again to a content of $0.98 \mu \mathrm{g} \mathrm{g}^{-1} \mathrm{FW}$. Throughout the development process, the $\mathrm{ZT}$ content remained at a low level (Fig. 2c). The abscisic acid (ABA) content increased sharply and reached a peak value of $124.93 \mu \mathrm{g} \mathrm{g}^{-1} \mathrm{FW}$ at the maturity stage (Fig. $2 \mathrm{~d}$ ). The peak of these three hormones in the process of seed develop-

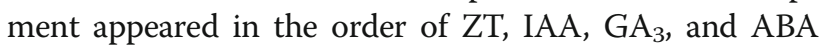
(Fig. 2e). Among the four phytohormones, their proportions were different at different stages of seed development (Fig. 2f).

\section{Nutrient accumulation at different stages of seed development}

As shown in Fig. 3, the four following components were quantified, namely, rutin, starch, total proteins and soluble sugars. The content of rutin increased linearly after anthesis and notably reached a peak value of 12055.78 $\mu \mathrm{g} \mathrm{g}^{-1}$ dry weight (DW) at the mature stage (Fig. 3a).
The content of starch rapidly increased during the transition to green fruit stage and reached a maximum of $167.40 \mathrm{mg} \mathrm{g}^{-1} \mathrm{DW}$, after which it decreased linearly to a content of $102.53 \mathrm{mg} \mathrm{g}^{-1}$ DW (Fig. 3b). The content of total protein gradually increased after anthesis, notably from the discoloration stage to the initial maturity stage, and reached a maximum of $4.64 \mathrm{mg} \mathrm{g}^{-1} \mathrm{FW}$ at the initial maturity stage, after which it started to drop rapidly to a content of $4.40 \mathrm{mg} \mathrm{g}^{-1} \mathrm{FW}$ (Fig. 3c). The soluble sugar linearly increased from the young fruit stage to the discoloration stage and reached a peak value of $0.21 \mathrm{mg} \mathrm{g}^{-1}$ DW at the discoloration stage. After a slight drop, it started to rise again to a content of $0.18 \mathrm{mg} \mathrm{g}^{-1}$ DW (Fig. 3d). Among the four nutrients, starch dominated with relatively high proportions at the different stages of seed development (Fig. 3e).

\section{Correlation between physiological and developmental changes in tartary buckwheat seeds}

The linear relationship between seed maturity and physiological changes were analyzed. The results showed that total protein, ZT and ABA were significantly correlated with seed maturity, and the absolute values of the correlation coefficients were all greater than 0.94 (Fig. 4). Seed size (transverse diameter $\times$ longitudinal diameter) was positively related to starch, with a correlation coefficient value of 0.906 . Longitudinal diameter was significantly 

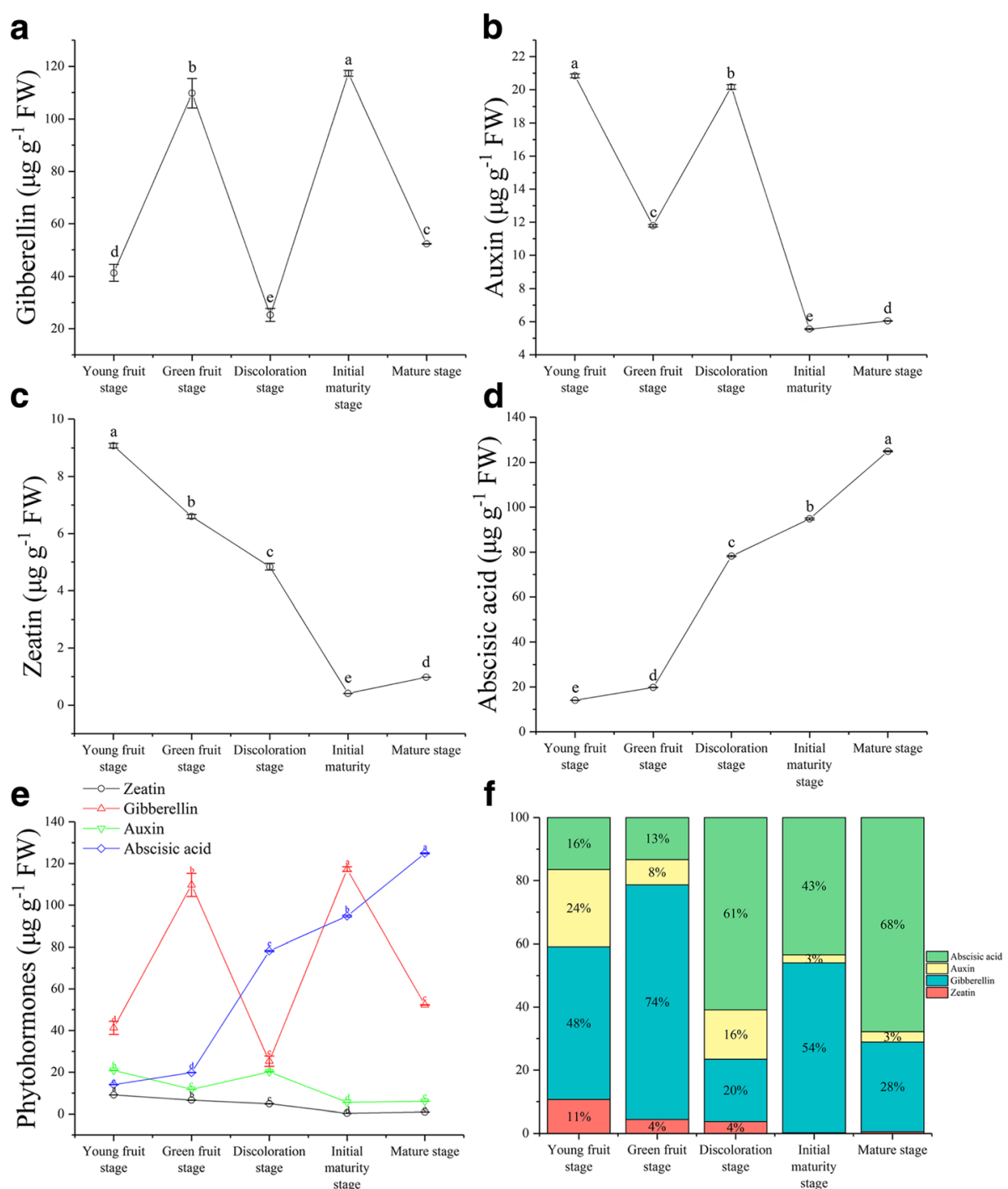

Fig. 2 Changes in the levels of $G A_{3}, I A A, Z T$, and $A B A$ during seed development in tartary buckwheat. a-d Changes of $G A_{3}, I A A, Z T$, and $A B A$ content during seed development in tartary buckwheat, respectively. e $\mathrm{GA}_{3}$ (red line), IAA (green line), ZT (black line), and ABA (blue line) content at different developmental stages. $\mathbf{f}$ Phytohormone ratio during seed development in tartary buckwheat. Error bars were obtained from three measurements for (a), (b), (c), (d) and (e). Small letter(s) above the bars indicate significant differences $(a=0.05$, LSD) among treatments

related to starch, and the correlation coefficient was 0.972 . Seed weight was positively related to soluble sugar, with a correlation coefficient value of 0.917 (Fig. 4). Total protein was significantly related to $\mathrm{ABA}$ and $\mathrm{ZT}$, and the absolute values of the correlation coefficients were greater than 0.93. The total protein was positively related to ABA and was negatively related to $\mathrm{ZT}$. ZT was negatively related to $\mathrm{ABA}$, and the absolute value of the correlation coefficient was 0.922 . However, rutin, $\mathrm{GA}_{3}$, IAA, seed size, seed weight and DPA were not significantly correlated (Fig. 4).

\section{Transcriptomic profiles during seed development}

To comprehensively assess the change in the transcript profile, we analyzed RNAs from three seed developmental stages $(13,19$, and $25 \mathrm{DPA})$. The raw reads of 9 samples ranged from 51 to 63 million, with an error rate of approximately 0.02 , resulting in 7.61-8.84 G of clean bases (Additional file 1: Table $\mathrm{S} 1$ ). In exon region, intron region and intergenic region, the reading rates of mapping were 97.4, 0.68, and $1.93 \%$, respectively (Fig. 5a). Approximately 91.94\% of total reads were mapped to the reference genome. Among them, the unique map reads accounted for $86.0 \%$ of the total reads, and multiple map reads accounted for 5.94\% (Fig. 5b, Additional file 1: Table S2). The overall quality of the RNA-seq data was evaluated by correlation analysis between samples and principal component analysis (PCA) of biological replicates (Fig. 5d). The correlation coefficients between all biological replicate 


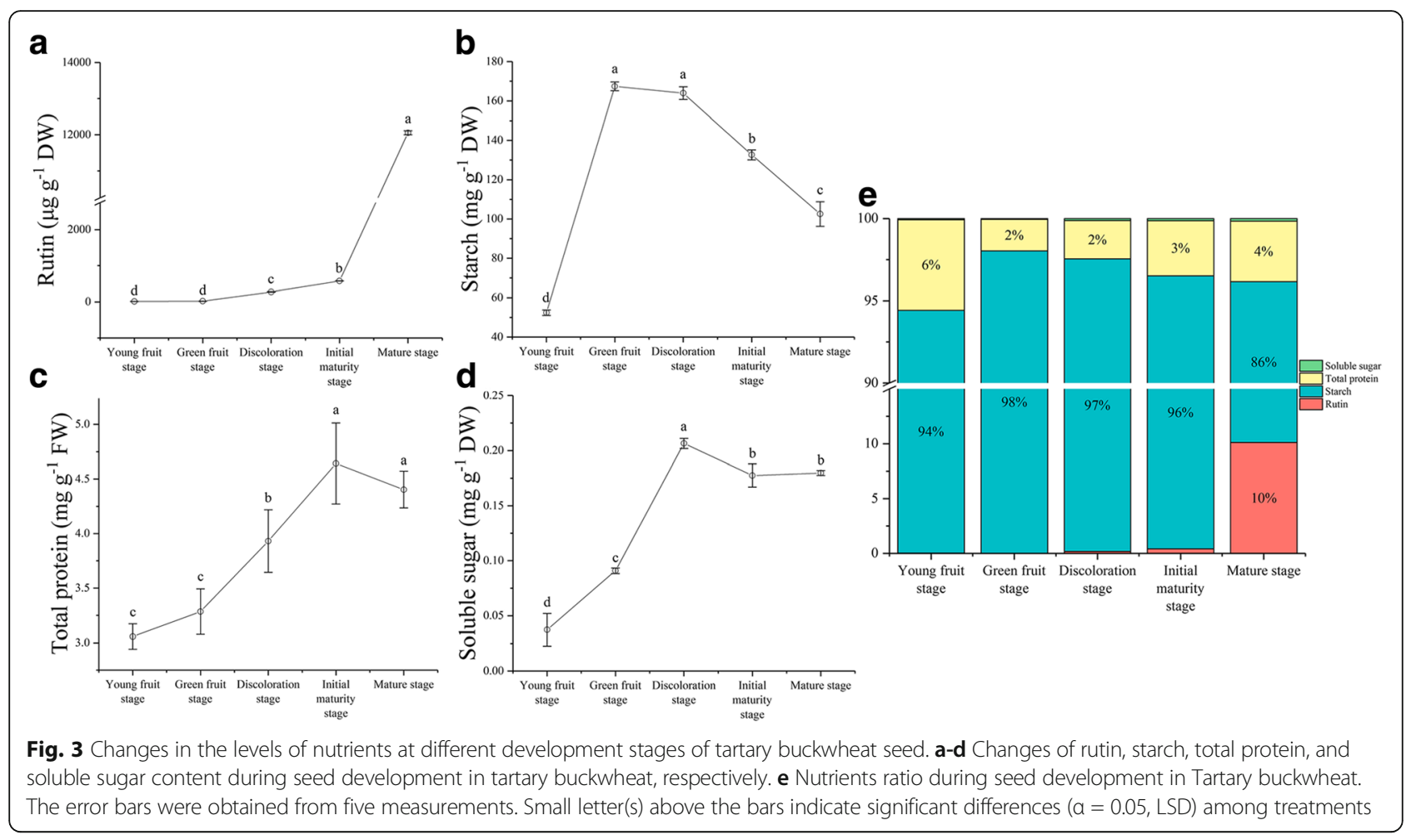

samples were greater than 0.90 (Fig. 5c, Additional file 1: Table S3). Three biological replicates, especially at 19 DPA, were significantly clustered, indicating that the expression patterns in the sample had high similarity and that the sequencing data could be used to analyze the differential expression of the genes. The expression levels of the genes were estimated by the fragments per kilobase of transcripts per million mapped fragments (FPKM) [13]. The level of gene expression showed a similar trend among the three samples, and the expression level of most genes was relatively low (Fig. 5e).

\section{Analysis of differentially expressed genes during seed development}

To understand gene expression during tartary buckwheat seed development (13, 19, and 25 DPA), we compared and analyzed the DEGs in two pairs of samples (13 DPA vs 19 DPA, 19 DPA vs 25 DPA). A total of 9 765 DEGs were divided into 6 groups (Fig. 6a). Similarly, they could be divided into two broad categories according to their expression patterns. The first category contained groups III, IV and VI, with upregulated genes. The second category contained groups I, II, and V, in which the genes showed downregulation patterns. In detail, groups III and IV showed a gradual upward trend during the seed development stage. The genes in group VI were upregulated at 25 DPA compared with those at 13 DPA and 19 DPA. Groups I, II and V showed a decreasing trend at the stage of seed development (Fig. 6b). We found significant differences in gene expression during seed development and detected 1 529 DEGs (791 downregulated and 738 upregulated) at 13 DPA vs 19 DPA and 5998 DEGs (2 337 downregulated and 3661 upregulated) at 19 DPA vs 25 DPA (Fig. 6c). Besides, the expression profiles of 20 genes in the 13DPA, 19DPA, and 25 DPA, based on RT-qPCR, were consistent with the RNA-seq results with a Pearson correlation coefficient of $0.31(\mathrm{P}<0.05)$ (Additional file 1: Table S6). To characterize the expression pattern of genes during tartary buckwheat seed development, we analyzed the overlapping DEGs in different sample combinations and measured 665 DEGs in the three samples (Fig. 6d); in the comparisons of 13 DPA vs 19 DPA and 19 DPA vs 25 DPA, there were 864 and 5244 DEGs, respectively (Fig. 6d).

\section{Expression patterns of DEGs related to phytohormones during seed development}

Phytohormones affect gene expression and transcription levels, cellular division, and plant growth [14]. Therefore, we identified the DEGs related to phytohormones. The results showed that 277 genes were associated with four major phytohormones, including ABA (35 genes), IAA (185 genes), ZT (20 genes) and $\mathrm{GA}_{3}$ (37 genes) (Additional file 1: Table S4). These genes are related to all aspects of phytohormone homeostasis, including biosynthesis (33 genes), metabolism (15 genes), receptors (26 genes), responses (3 genes), signal transduction (178 genes) and 


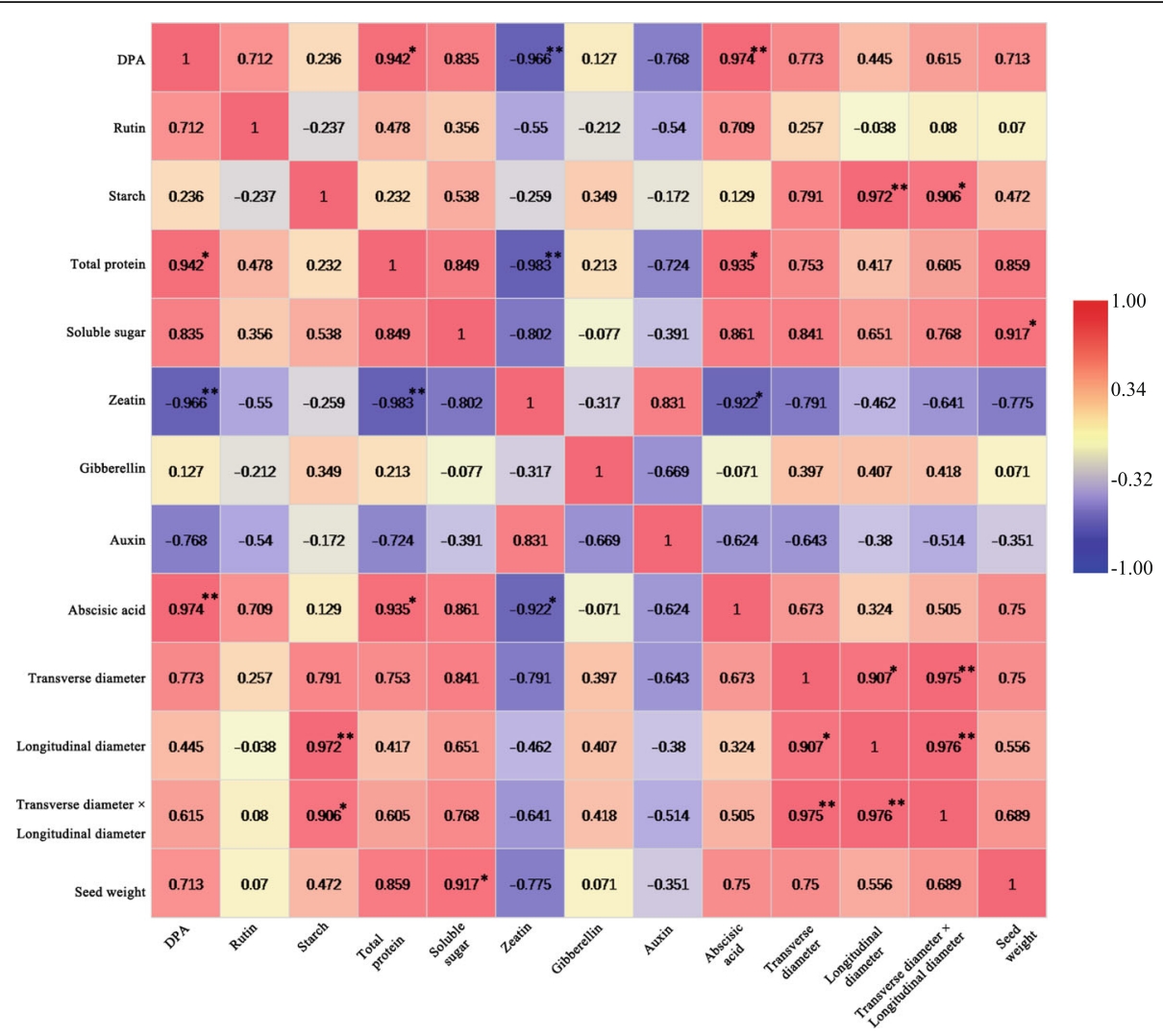

Fig. 4 The correlation between physiological changes and seed maturity during seed development (7 DPA to 30 DPA). Digit: Pearson's correlation coefficient; Red: positively correlated; Blue: negatively correlated. * and ** indicate significant correlation at 0.05 and 0.01 levels, respectively

transportation (22 genes) (Table 1). The number of them found in a complex regulatory network of seed development are presented on Fig. 7a. We detected 17 DEGs (9 downregulated DEGs and 8 upregulated DEGs) in 13 DPA vs $19 \mathrm{DPA}$ and 57 DEGs (16 downregulated DEGs and 41 upregulated DEGs) in 19 DPA vs 25 DPA (Fig. 7i). In 13 DPA vs 19 DPA, the DEGs were mostly enriched in 4 KEGG pathways in the KEGG database (False discovery rate $($ FDR $)<0.05)$ (Fig. 7b). However, a Gene Ontology (GO) analysis indicated that DEGs were not significantly enriched (FDR < 0.05) (Fig. 7d). In 19 DPA vs 25 DPA, the DEGs were mostly enriched in 6 KEGG pathways in the KEGG database (FDR < 0.05) (Fig. 7c). A GO analysis indicated that 27 terms involving biological process (Fig. 7e), 9 terms involving cellular component (Fig. $7 \mathrm{f}$ ), and 3 terms involving molecular function (Fig. $7 \mathrm{~h}$ ) were significantly enriched (FDR < 0.05) (Fig. 7e).

\section{Expression patterns of DEGs related to flavonoids during seed development}

Tartary buckwheat is rich in flavonoids, including rutin and other flavonoids [15]. As a result, 47 genes were annotated as related to flavonoids. Among these genes, the DEGs included 3 phenylalanine ammonia-lyase (PAL), 4 chalcone synthase (CHS), 2 chalcone isomerase (CHI), 8 flavonol synthase (FLS), 6 flavonoid 3',5'-hydroxylase, 14 UDP-glucose flavonoid, and 10 flavonoid 3'-monooxygenase (Additional file 1: Table S5). According to their level of expression by developmental stage, they have at least one highly expressed member at all developmental stages. In 13 DPA vs 19 DPA, the DEGs were mostly enriched in phenylalanine metabolism pathways (ath00360) in the KEGG database (FDR < 0.05) (Additional file 1: Figure S1b), including 1 DEG (Table 2). In 19 DPA vs 25 DPA, 4 DEGs were mostly enriched in flavonoid biosynthesis pathways (ath00941) in the KEGG database (FDR < 0.05) (Additional file 1: Figure S1c, Table 2). These DEGs showed a higher expression in the flavonoid biosynthesis pathway from 13 DPA to 19 DPA (Additional file 1: Figure S1a).

\section{Expression patterns of DEGs related to starch during seed development}

Starch is among the main nutrients in tartary buckwheat and dominates with relatively high proportions [16]. We identified 20 genes related to starch, including 5 soluble starch synthase, 9 starch synthase, and 6 granule-bound starch synthase genes, all of which belong to the glycosyltransferase 1 family (Table 3 ). 


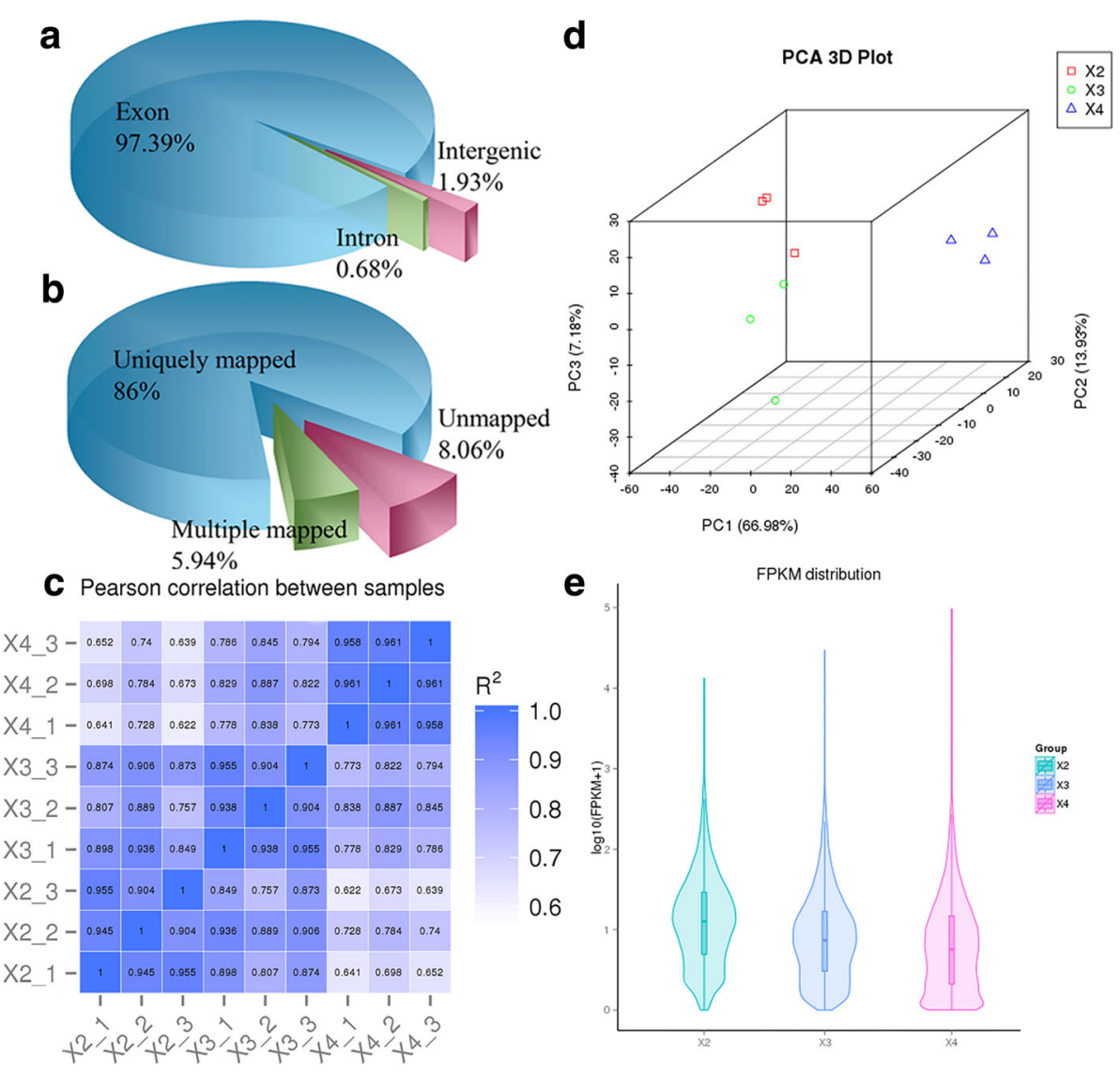

Fig. 5 Distribution and quality of reads in different samples. a Distribution of overall mapped reads for all samples in the different regions. $\mathbf{b}$ Overall results of paired-end reads (PEs) for all samples mapped to the reference genome. $\mathbf{c}$ Correlation between samples. $\mathbf{d}$ Principal component analysis (PCA) among biological replicates. e Gene expression distribution (FPKM) in three samples

In 13 DPA vs 19 DPA, no genes were differentially expressed (FDR < 0.05). In 19 DPA vs 25 DPA, 3 DEGs were involved in starch biosynthesis (Additional file 1: Figure S2).

\section{Expression patterns of DEGs related to storage proteins during seed development}

On the basis of differential solubility, storage proteins include globulin, albumin, glutelin, and prolamin [17]. As such, of the 21 genes, 18 are DEGs related to storage proteins, including 16 related to globulin and 2 related to glutelin (Additional file 1: Figure S3a). In 13 DPA vs 19 DPA, a GO analysis showed that the DEGs were significantly enriched in the molecular function term "nutrient reservoir activity" (GO:0045735) (Additional file 1: Figure S3b, c). In 19 DPA vs 25 DPA, the GO analysis showed that the DEGs were significantly enriched in the molecular function terms including nutrient reservoir activity (GO:0045735) and acireductone dioxygenase [iro$\mathrm{n}$ (II)-requiring] activity (GO:0010309) (Additional file 1: Figure S3d, e).

\section{Discussion}

Correlation analysis between embryo cell enlargement and phytohormones during the development of buckwheat seed

The role of auxin in cell enlargement and the development of plant organs have been fully confirmed $[18,19]$, and the seed is no exception. In our study, the content of IAA decreased gradually as a fluctuation curve during the development of buckwheat seed (Fig. 2b), and we identified 18 DEGs in transcriptome; these DEGs were enriched in the plant hormone signal transduction pathway (ath04075) and may be related to cell enlargement (Fig. 8m). Most of these DEGs were downregulated from 13 DPA to 25 DPA. FtPinG0007643300.01, FtPinG0 003888700.01, and FtPinG0006908400.01 were matched to the amino acid/polyamine transporter 2 family, whose members are located on the plasma membrane (Fig. 81) and are involved in proton-driven auxin influx. In addition, 7 DEGs were matched to the Aux/IAA family and located in the nucleus. Aux/IAA proteins are short-lived transcription factors and act as inhibitors of early auxin response genes 


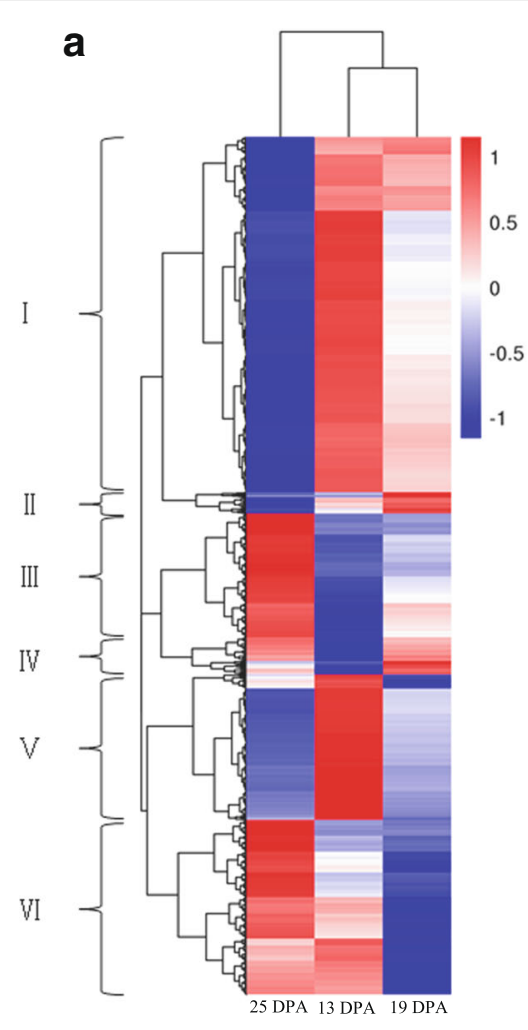

\section{b}
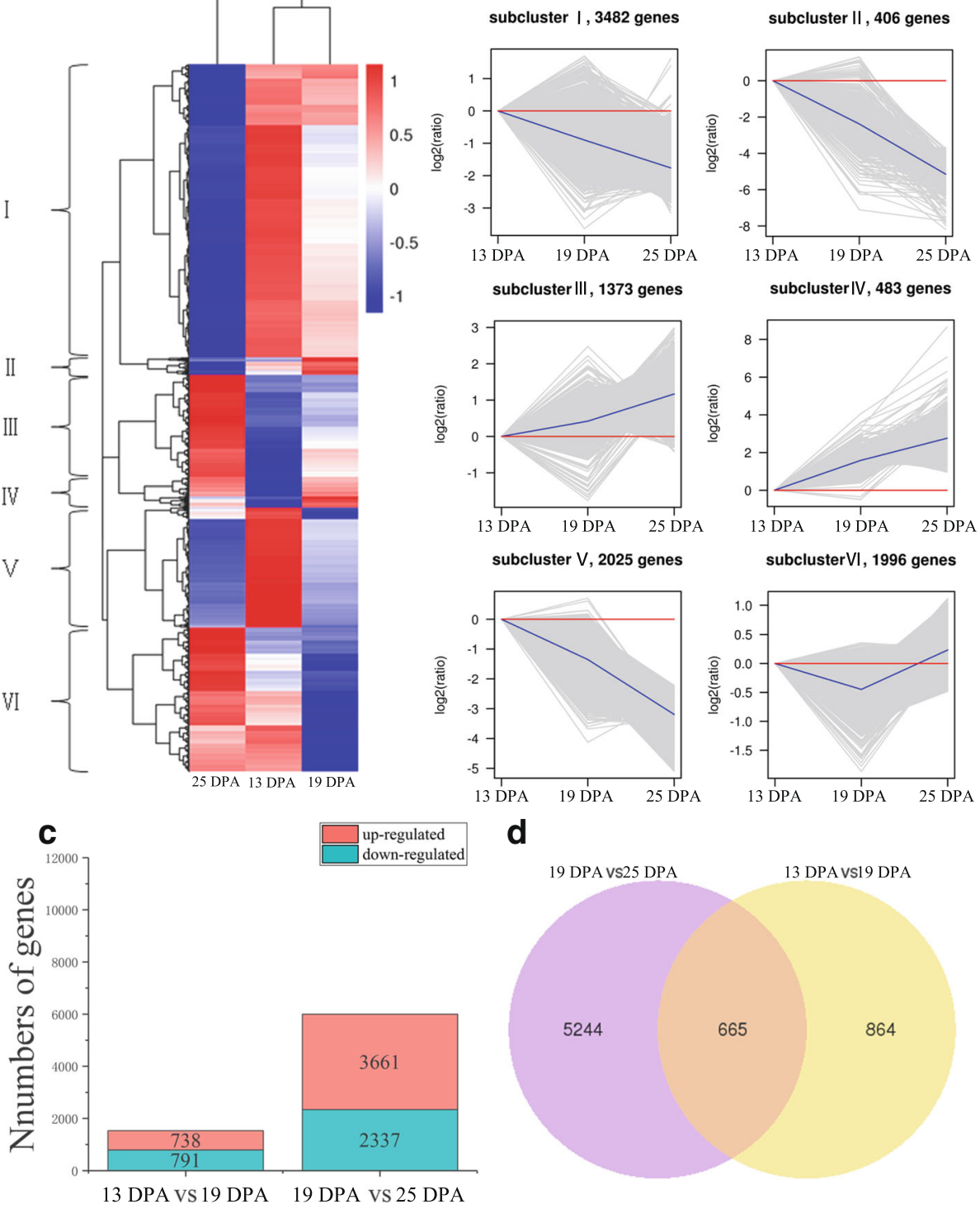

d

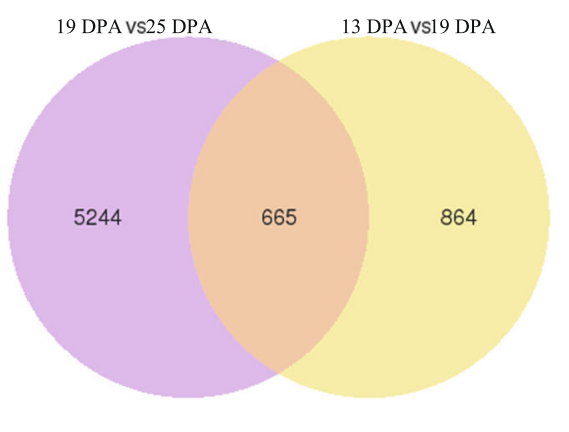

Fig. 6 DEGs in three samples. a Heat map of scaled FPKM values in three samples 13 DPA, 19 DPA, and 25 DPA. Red: high expression; Blue: low expression. $\mathbf{b}$ Expression profile of six clusters correspondance to the Hierarchical cluster result. c Number of up- and down-regulated DEGs in two sample pairs 13 DPA vs 19 DPA, and 19 DPA vs 25 DPA. d Venn diagram of the numbers of expressed genes in sample pairs 13 DPA vs 19 DPA, and 19 DPA) vs 25 DPA

at low concentrations of auxin [20]. Novel02020, FtPinG0000807700.01, and FtPinG0008443000.01 were matched to the ARF family and are located in the nucleus (Fig. 81). Auxin response factors (ARFs) are transcription factors that specifically bind to the DNA sequence 5'-TGTCTC-3' found in auxin-responsive promoter elements (AuxREs) [21-23]. Five DEGs were matched to the ARG7 family whose members are located in the nucleus, and these DEGs may play a role in apical hook development [24]. According to our previous research results and related research, during the development of buckwheat seeds we hypothesize that the extent of seed embryo cell enlargement gradually decreased, and the final embryo cell size was no longer increased and tended to stabilize. To test this hypothesis, we compared the size of seed embryo cells from 7 DPA to 30 DPA. However, the extent of seed embryo cell enlargement gradually increased (Fig. 8k). In fact, the decrease in auxin concentration may not be enough to stop cell enlargement. Auxin plays a role in initiation or acts as a 'on' switch via secondary regulators. Only lowering the auxin concentration (that is, removing the 'on' switch) does not reduce other phytohormones. 
Table 1 Genes related to phytohormones during seed development of tartary buckwheat

\begin{tabular}{lllllllc}
\hline & Number of DEGs & Biosynthesis & Metabolism & Receptor & Response & Signal transduction & Transportion \\
\hline ABA & 35 & 0 & 8 & 16 & 0 & 11 & 0 \\
IAA & 185 & 0 & 0 & 6 & 0 & 157 & 22 \\
ZT & 20 & 13 & 7 & 0 & 0 & 0 & 0 \\
GA $_{3}$ & 37 & 20 & 0 & 4 & 3 & 10 & 0 \\
Total & 277 & 33 & 15 & 26 & 3 & 178 & 22 \\
\hline
\end{tabular}

Considering this hypothesis, a second 'on' switch may be required to accurately determine the time frame for cell enlargement [25-30]. It was found that the content of ABA is different in embryogenic cell clusters, globular embryos, torpedo embryos, and cotyledon embryos [31].
During embryonic development, ABA affects starch biosynthesis and carbohydrate absorption and directly regulates or initiates the biosynthesis of protein [32], DNA, and mRNA [33], all of which have an effect on the enlargement of embryonic cells [34]. According to these
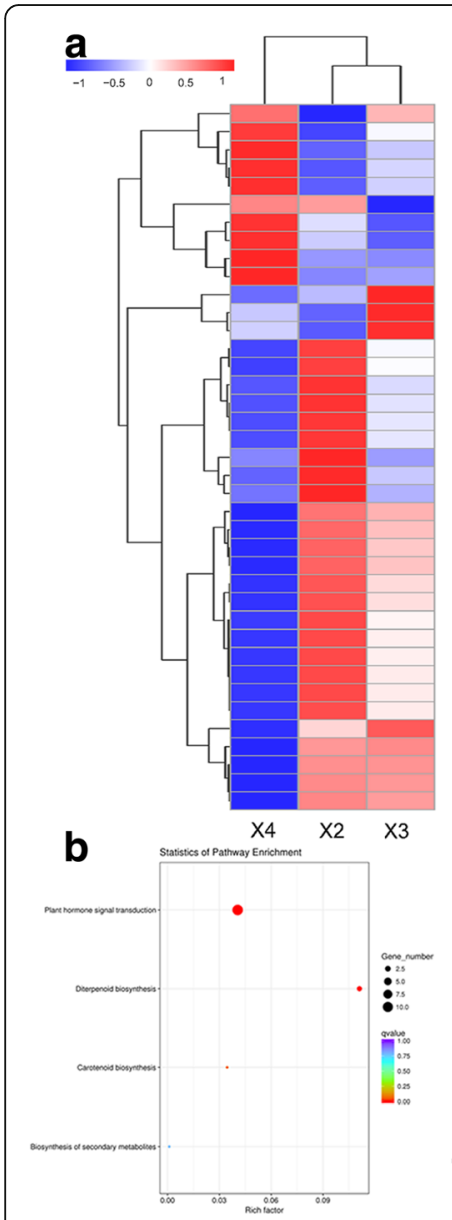

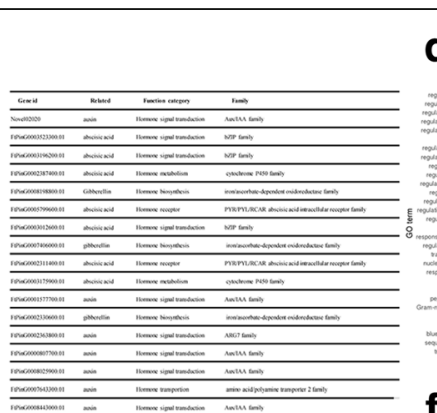

\section{d}
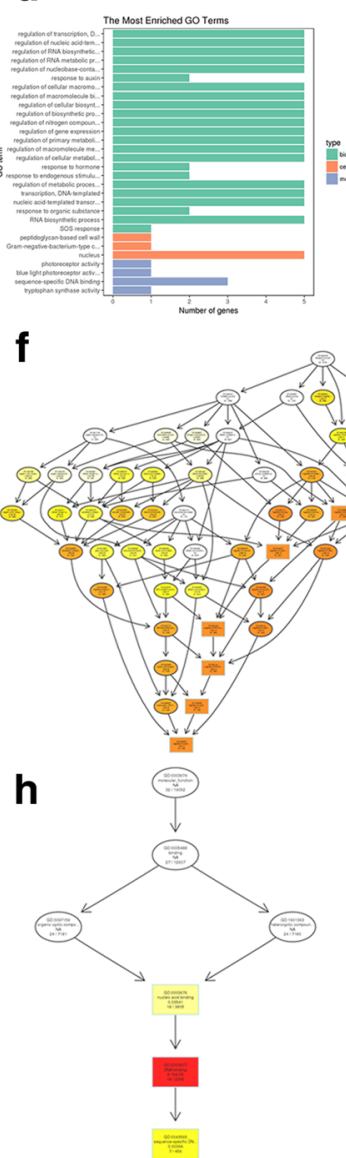

e

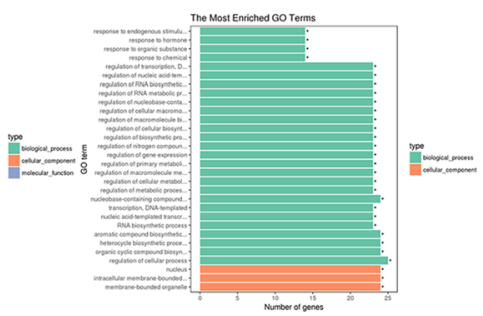

9

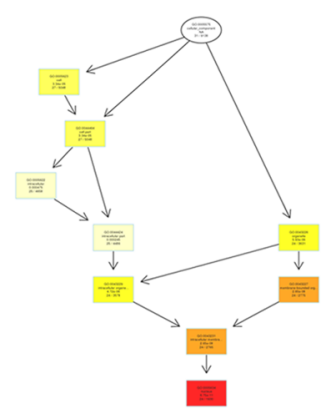

i

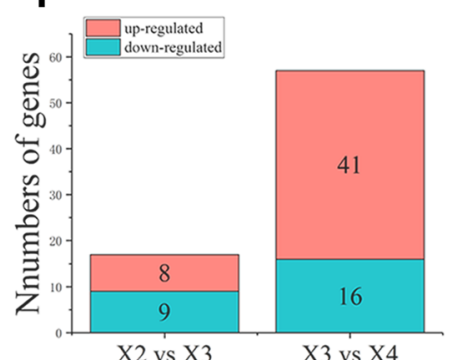

Fig. 7 DEGs related to phytohormones in three samples. a Hierarchical cluster of the DEGs related to phytohormones in X2 (13 DPA), X3 (19 DPA), and X4 (25 DPA). Red: high expression; Blue: low expression. b Scatterplot of KEGG pathway enrichment in X2 (13 DPA) vs X3 (19 DPA) (FDR< 0.05). c Scatterplot of KEGG pathway enrichment in X3 (19 DPA) vs X4 (25 DPA) (FDR< 0.05). Rich factor is the ratio of the number of DEGs to the number of background genes in a KEGG pathway. $\mathbf{d}$ GO classification of DEGs in X2 (13 DPA) vs X3 (19 DPA) (FDR< 0.05). e GO classification of DEGs in X3 (19 DPA) vs X4 (25 DPA) (FDR< 0.05). The top 30 enriched GO classifications are listed. Stars above bars indicate the amounts of differentially expressed genes are significantly higher or lower than the amounts of genes in random samples from the GO classification of all genes. $\mathbf{f}$ DEGs related to phytohormones enriched biological processes in X3 (19 DPA) vs X4 (25 DPA). g DEGs related to phytohormones enriched cellular component in X3 (19 DPA) vs X4 (25 DPA). $\mathbf{h}$ DEGs related to phytohormones enriched molecular function in X3 (19 DPA) vs X4 (25 DPA). The different color frames indicate the extent of significance. Yellow: significant; Red: extremely significant. i Number of up- and down-regulated DEGs in two sample pairs X2 (13 DPA) vs X3 (19 DPA), and X3 (19 DPA) vs X4 (25 DPA) 
Table 2 Differentially expressed genes related to in flavonoid biosynthesis pathways during seed development of tartary buckwheat

\begin{tabular}{|c|c|c|c|c|c|c|c|c|c|}
\hline Gene id & Related & $\begin{array}{l}\text { Function } \\
\text { category }\end{array}$ & Family & Stage & Readcount & Readcount & Log $^{2}$ FoldChange & Padj & Significant \\
\hline FtPinG0008236900.01 & PAL & $\begin{array}{l}\text { Phenylpropanoid } \\
\text { metabolism }\end{array}$ & $\begin{array}{l}\text { PAL/histidase } \\
\text { family }\end{array}$ & $\begin{array}{l}X 2 \text { vs } \\
\times 3\end{array}$ & 2444.836885 & 6450.386734 & -1.3996 & 0.00817 & DOWN \\
\hline FtPinG0006907000.01 & FLS & $\begin{array}{l}\text { Flavonoid } \\
\text { biosynthesis }\end{array}$ & $\begin{array}{l}\text { iron/ascorbate- } \\
\text { dependent } \\
\text { oxidoreductase } \\
\text { family }\end{array}$ & $\begin{array}{l}X 3 \text { vs } \\
X 4\end{array}$ & 530.8399528 & 81.48645982 & 2.7036 & 0.024921 & UP \\
\hline FtPinG0008131000.01 & $\mathrm{CHS}$ & $\begin{array}{l}\text { Flavonoid } \\
\text { biosynthesis }\end{array}$ & $\begin{array}{l}\text { chalcone/stilbene } \\
\text { synthases family }\end{array}$ & $\begin{array}{l}X 3 \text { vs } \\
X 4\end{array}$ & 8286.397285 & 871.1066818 & 3.2498 & 0.00007048 & UP \\
\hline FtPinG0006907100.01 & FLS & $\begin{array}{l}\text { Flavonoid } \\
\text { biosynthesis }\end{array}$ & $\begin{array}{l}\text { iron/ascorbate- } \\
\text { dependent } \\
\text { oxidoreductase } \\
\text { family }\end{array}$ & $\begin{array}{l}X 3 \text { vs } \\
X 4\end{array}$ & 8600.271627 & 2007.552066 & 2.0989 & $1.5955 \mathrm{E}-06$ & UP \\
\hline FtPinG0002790600.01 & $\mathrm{CHI}$ & $\begin{array}{l}\text { Flavonoid } \\
\text { biosynthesis }\end{array}$ & $\begin{array}{l}\text { chalcone } \\
\text { isomerase family }\end{array}$ & $\begin{array}{l}X 3 \text { vs } \\
X 4\end{array}$ & 1641.630481 & 287.3719632 & 2.5141 & 0.00007764 & UP \\
\hline
\end{tabular}

reports, embryonic cells continuously biosynthesize and absorb nutrients, resulting in an enlarged size under the influence of ABA. This fact may reinforce the idea of ABA as the second switch for cell enlargement. Therefore, we speculate that the increase in and prolongation of $\mathrm{ABA}$ content from 7 DPA to 30 DPA may be related to the enlargement of seed embryo cells. In the end, via the content of $\mathrm{ABA}$, we confirmed that the content increased during the development of buckwheat seeds, which is consistent with the increase in seed embryo cells (Fig. 8k).

\section{Correlation analysis between dormancy and phytohormones during the development of buckwheat seed}

Dormancy occurs during seed development, and seeds are scattered from the parent plant in a dormant state [35]. ABA is a positive regulator of seed dormancy induction. Transgenic tobacco plants expressing anti-ABA antibody lack ABA, and the seeds are not dormant [36]. Overexpression of $\mathrm{ABA}$ biosynthesis genes can increase the content of ABA in seeds, thus promoting seed

Table 3 Genes related to starch synthesis (or metabolism) during seed development

\begin{tabular}{|c|c|c|c|c|c|c|}
\hline Gene id & Related & Function category & Family & X2_fpkm & X3_fpkm & X4_fpkm \\
\hline FtPinG0002952000.01 & Starch synthase & Starch biosynthesis & glycosyltransferase 1 family & 0 & 0.04209211 & 0 \\
\hline FtPinG0001526900.01 & Starch synthase & Starch biosynthesis & glycosyltransferase 1 family & 0.086131576 & 0 & 0 \\
\hline FtPinG0005431900.01 & Starch synthase & Starch biosynthesis & glycosyltransferase 1 family & 0 & 0 & 0 \\
\hline FtPinG0000359400.01 & Starch synthase & Starch biosynthesis & glycosyltransferase 1 family & 57.63315519 & 328.8316391 & 5.916537825 \\
\hline FtPinG0007470100.01 & Starch synthase & Starch biosynthesis & glycosyltransferase 1 family & 13.26934373 & 7.27664449 & 1.747617671 \\
\hline FtPinG0009838000.01 & Starch synthase & Starch biosynthesis & glycosyltransferase 1 family & 451.7534424 & 367.1546165 & 1.968141961 \\
\hline FtPinG0006332800.01 & Starch synthase & Starch biosynthesis & glycosyltransferase 1 family & 20.95952758 & 7.358124306 & 9.756228183 \\
\hline FtPinG0008723400.01 & Starch synthase & Starch biosynthesis & glycosyltransferase 1 family & 12.73350096 & 8.974233732 & 13.93766394 \\
\hline FtPinG0007775600.01 & Starch synthase & Starch biosynthesis & glycosyltransferase 1 family & 42.32919719 & 32.13196216 & 36.80551599 \\
\hline FtPinG0000021600.01 & Starch synthase & Starch biosynthesis & glycosyltransferase 1 family & 1.21734049 & 1.02004409 & 1.121365717 \\
\hline FtPinG0000380300.01 & Starch synthase & Starch biosynthesis & glycosyltransferase 1 family & 43.88186662 & 18.86806009 & 7.382532431 \\
\hline FtPinG0005565800.01 & Starch synthase & Starch biosynthesis & glycosyltransferase 1 family & 198.3185567 & 88.78020142 & 53.22576629 \\
\hline FtPinG0003226800.01 & Starch synthase & Starch biosynthesis & glycosyltransferase 1 family & 43.42372433 & 16.80944338 & 2.358411337 \\
\hline FtPinG0005368600.01 & Starch synthase & Starch biosynthesis & glycosyltransferase 1 family & 19.42316605 & 5.279935074 & 1.734551104 \\
\hline FtPinG0007534500.01 & Starch synthase & Starch biosynthesis & glycosyltransferase 1 family & 36.91435118 & 17.93308621 & 14.83261806 \\
\hline FtPinG0007419600.01 & Starch synthase & Starch biosynthesis & glycosyltransferase 1 family & 11.0790269 & 7.395763029 & 10.50993485 \\
\hline FtPinG0003227000.01 & Starch synthase & Starch biosynthesis & glycosyltransferase 1 family & 6.54592268 & 2.517975445 & 0.525192882 \\
\hline FtPinG0005939600.01 & Starch synthase & Starch biosynthesis & glycosyltransferase 1 family & 82.30668491 & 70.82139489 & 55.11481588 \\
\hline FtPinG0003590400.01 & Starch synthase & Starch biosynthesis & glycosyltransferase 1 family & 16.54472332 & 9.19667544 & 7.289004226 \\
\hline FtPinG0005109900.01 & Starch synthase & Starch biosynthesis & glycosyltransferase 1 family & 47.48674066 & 23.91252077 & 4.300813932 \\
\hline
\end{tabular}



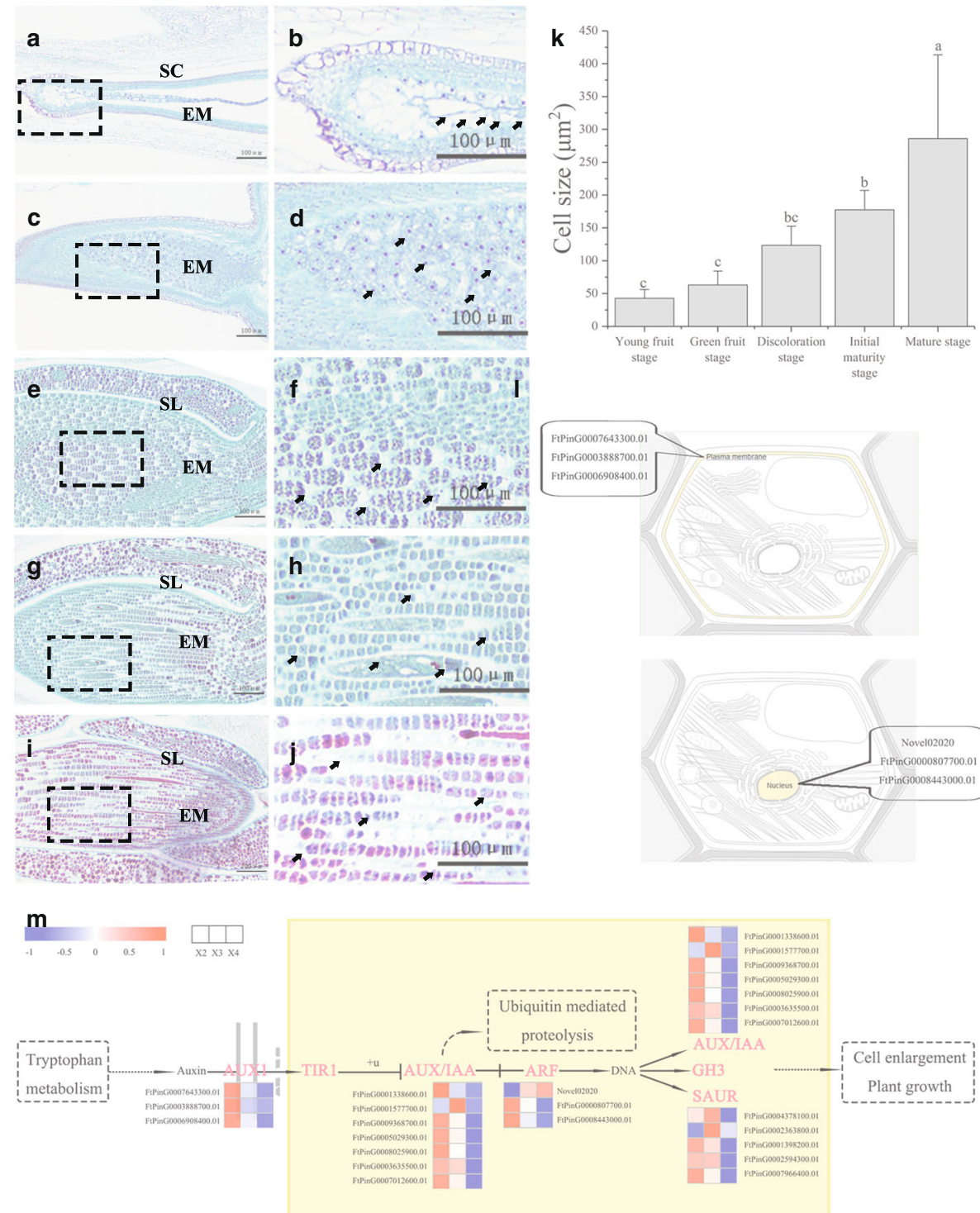

Fig. 8 Enlargement of embryo cell during tartary buckwheat seed development. $\mathbf{a}, \mathbf{c}, \mathbf{e}, \mathbf{g}$ and $\mathbf{i}$ Microscopic longitudinal sections of Tartary buckwheat seeds at 7, 13, 19, 25 and 30 DPA, respectively. $\mathbf{b}, \mathbf{d}, \mathbf{f}, \mathbf{h}$ and $\mathbf{j}$ are the enlarged view of the boxes in (a), (c), (e), (g) and (i), respectively. SC (Seed cover). EM

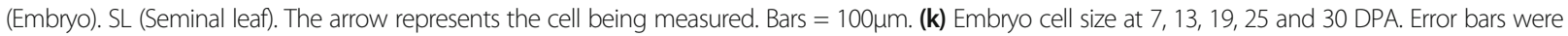
obtained from five measurements. Small letter(s) above the bars indicate significant differences ( $a=0.05, \mathrm{LSD})$ among treatments. (I) Subcellular location of DEGs related to embryo cell enlargement. This section provides information on the location in the cell (Graphics by Christian Stolte). $\mathbf{m}$ A simplified representation of the plant hormone signal transduction pathway of cell enlargement (adopted from the KEGG PATHWAY Database: http:// www.genome.jp/kegg/pathway.html) shows the following: auxin influx carrier (AUX1), transport inhibitor response 1 (TIR1), auxin-responsive protein IAA (AUX/IAA), auxin response factor (ARF), auxin responsive GH3 gene family (CH3), and SAUR family protein (SAUR). The expression value of each gene is colored in $\log _{10}($ FPKM) in three samples X2 (13 DPA), X3 (19 DPA), and X4 (25 DPA)

dormancy or delayed germination [37]. The biosynthesis of GA does not correlate with the establishment of primary dormancy [38]. However, $\mathrm{GA}_{3}$ plays important roles in the seed development of tomato, pea (Pisum sativum) and some Brassica plants, including fertilization, assimilation, embryonic growth, prevention of seed abortion, and fruit growth [39, 40]. A model of seed dormancy and germination was regulated by $A B A$ and $\mathrm{GA}_{3}$, and the complex phenomenon was explained by the response to environmental factors. The model was affected not only by many genes but also by plant hormones and environmental factors [41, 42]. According to this model, the biosynthesis of $\mathrm{ABA}$ and the signal of $\mathrm{GA}_{3}$ catabolism determine the dormancy of the seed, and the biosynthesis of $\mathrm{GA}_{3}$ and the signal of ABA catabolism determine the transformation of seeds to begin germinating. 
Our data show that the content of ABA was significantly related to seed development (Fig. 4). The content of ABA increased with the development of buckwheat seeds, especially during 13 DPA to 25 DPA (Fig. 2d), when a large amount of storage material accumulated (Fig. 3). Moreover, only 2 DEGs (FtPinG0002387400.01 and FtPinG0003 614600.01) that were involved in the oxidative degradation of ABA were downregulated during $19 \mathrm{DPA}$ to $25 \mathrm{DPA}$ (Fig. 7a). We also found the peak ABA/GA ratio occurred at 19 DPA (Fig. 9a). Based on the results we described earlier and those of related studies, we hypothesize that a large number of genes related to buckwheat seed dormancy are expressed from 19 DPA to 25 DPA. To test this hypothesis, we identified 11 transcriptome DEGs that were enriched in plant hormone signal transduction pathways (ath04075) and that were related to seed dormancy from 19 DPA to 25 DPA (Fig. 9c). Among them, the upregulated FtPinG0005799600.01 and FtPinG 0002311400.01 belong to the PYR/PYL/RCAR abscisic acid intracellular receptor family, whose members are located on the plasma membrane and in the nucleus [43] (Fig. 9b). FtPinG0005799600.01 was matched to the abscisic acid receptor (PYL4) gene, and FtPinG0002311400.01 was matched to the abscisic acid receptor (PYL10) gene; both are $\mathrm{ABA}$ receptors required for ABA-mediated responses, and when activated by $\mathrm{ABA}$, they inhibit the activity of group-A protein type $2 \mathrm{C}$ phosphatases (PP2Cs) [44-46]. In addition, the upregulated FtPinG0003523300.01, FtPinG00 03196200.01, and FtPinG0003012600.01 belong to the bZIP family, whose members are located in the nucleus (Fig. 9c). FtPinG0003523300.01 was matched to the protein ABSCISIC ACID-INSENSITIVE 5, which participates in the regulation of ABA-regulated gene expression during seed development and joins the embryonic normative element and the ABA-responsive element (ABRE) of the Dc3 gene promoter and the ABRE gene promoter in the $\mathrm{Em} 1$ and Em6 genes [47-53]. FtPinG0003196200.01 and FtPinG000 3012600.01 were matched to ABSCISIC ACID-INSENSITIVE 5-like protein 5, which is involved in the ABA response, acts as a positive component of glucose signal transduction, and specifically binds to the ABRE of the rd29B gene promoter [53-56]. As expected, a large number of genes related to buckwheat seed primary dormancy were expressed during 19 DPA to 25 DPA, and we identified good candidate genes related to buckwheat seed dormancy.

\section{Insights into the correlations between nutrient changes in and seed development}

Starch is the major component of buckwheat nutrients and accounts for more than $86 \%$ of nutrients (Fig. 3e). Starch causes seeds and other storage organs to expand and enlarge during seed development (Fig. 1a); thus, it plays an important role in the size, weight and quality of buckwheat

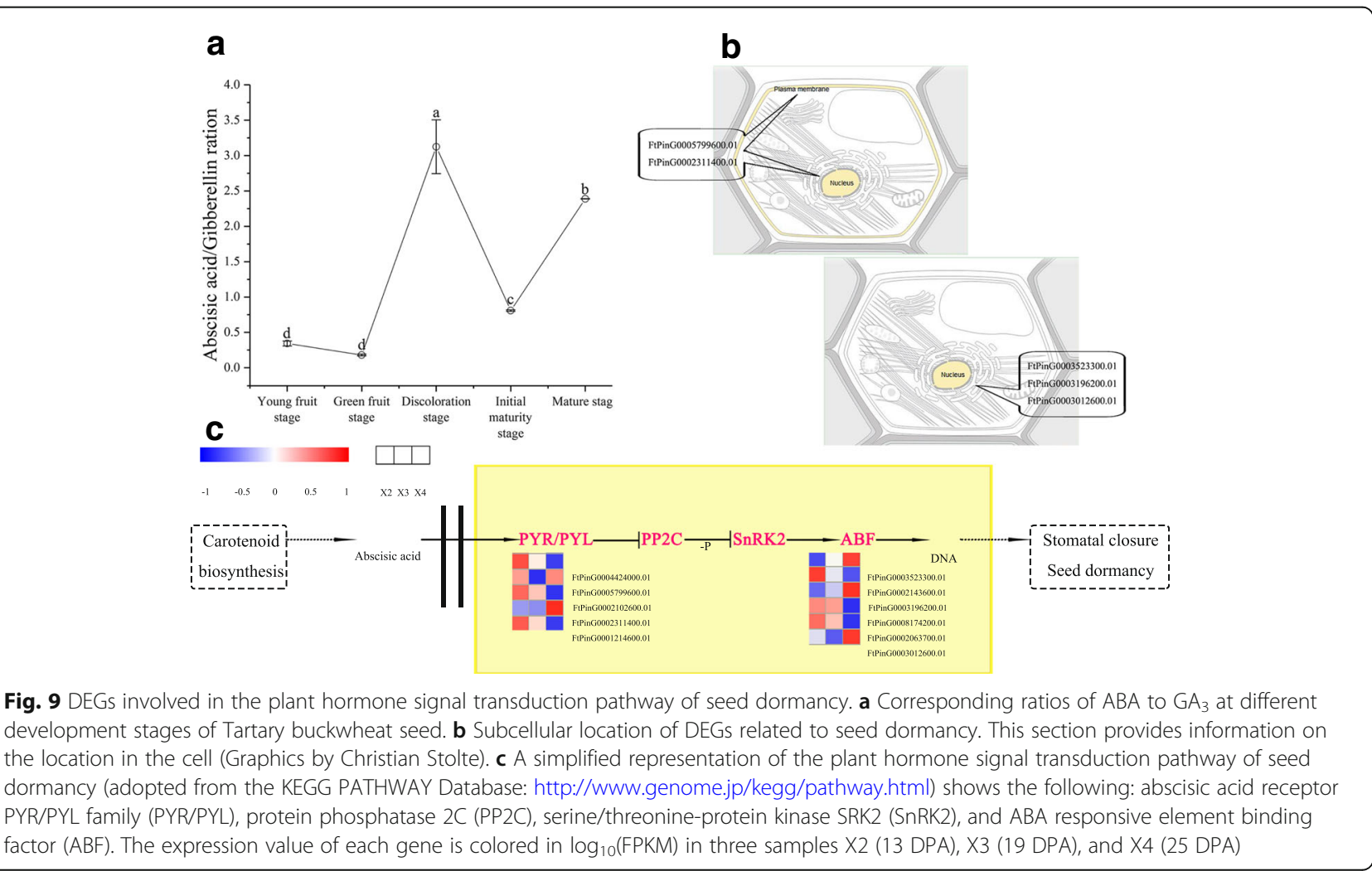


seeds during their development. In this study, starch was highly associated with seed size (especially the longitudinal diameter) during buckwheat seed development (Fig. 4). The seeds increased significantly during 7 DPA to 13 DPA (Figs 1b, 3b), which means buckwheat seeds and other storage organs expand and enlarge mainly during prophase (Fig. 1b). In our study, we identified 3 DEGs related to starch during seed development (Additional file 1: Figure S2). Among them, FtPinG0008723400.01 was matched to the SS4 gene, which is located in the chloroplast (Fig. 10i). SS4 may be involved in the initiation of starch granule formation and may play a regulatory role in controlling starch accumulation in plastids [57-59]. In our data, the expression of FtPinG0008723400.01 was upregulated only during 19 DPA to 25 DPA, and it is plausible to infer that a large number of starch granules were formed at the same time. To test this hypothesis, we compared the starch granule numbers and size at $13 \mathrm{DPA}$, 19 DPA and 25 DPA (Fig. 10). As expected, there was noticeable difference in starch granules numbers and size from 19 DPA to 25 DPA (Fig. 10g, h), suggesting that FtPinG0008723400.01 may be involved in the increase in starch granules and may play a regulatory role in controlling starch accumulation in starch storage cells. In addition, the expansion of starch storage cells was mainly due to the increase in starch granules size, not the increase in the number of starch granules (Fig. 10g, h). Furthermore, we found that FtPinG0007470100.01 and FtPinG000 0380300.01 were matched to the WAXY gene, which is located in the chloroplast (Fig. 10i). This protein is involved in the biosynthesis of starch and is a part of glycan biosynthesis, which is responsible for the synthesis of the amylose component of starch. In our study, the expression of these genes was downregulated, and starch also decreased

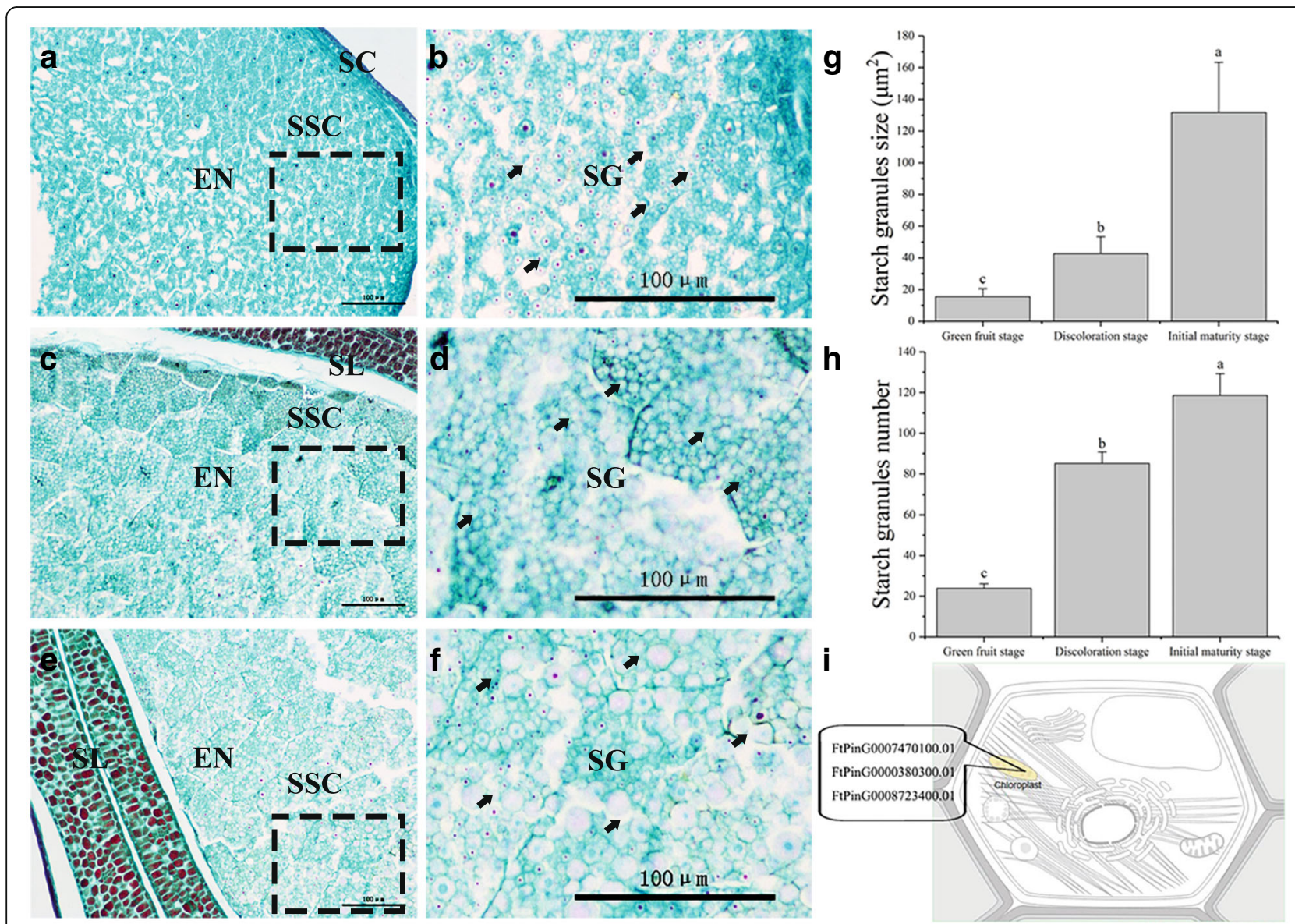

Fig. 10 Comparison of starch granules morphology during different development stages of tartary buckwheat seed. a, c, and e Microscopic longitudinal sections of Tartary buckwheat seeds at 13, 19, and 25 DPA, respectively. b, d, and $\mathbf{f}$ are the enlarged view of the boxes in (a), (c), and (e), respectively. SC (Seed cover). EN (Endosperm). SL (Seminal leaf). SSC (Starch storage cells). SG (Starch granules). The arrow represents the cell being measured. Bars $=100 \mu \mathrm{m}$. $\mathbf{g}$ Starch granules size at 13 DPA, 19 DPA, and 25 DPA. $\mathbf{h}$ Starch granules number (Average of total starch granules in each starch storage cell) at 13 DPA, 19 DPA, and 25 DPA. Error bars were obtained from five measurements for (g) and (h). Small letter(s) above the bars indicate significant differences $(a=0.05$, LSD) among treatments. i Subcellular location of DEGs related to starch biosynthesis. This section provides information on the location in the cell (Graphics by Christian Stolte) 
significantly from 19 DPA to 25 DPA (Fig. 3b). Hence, FtPinG0008723400.01, FtPinG0007470100.01 and FtPinG0 000380300.01 are good candidate genes related to buckwheat starch biosynthesis during seed development.

Total protein, including proenzymes, enzymes, and metabolic regulatory substances, is the second most dominant component of buckwheat nutrients, and storage protein accounts for $2 \sim 6 \%$ of nutrients (Fig. 3e). During seed development, many proteins are regulatory factors that are involved in metabolism $[60,61]$, so protein is the most active part of buckwheat nutrients. In our data, the total protein content was strongly associated with seed development (Fig. 4). The total protein content increased significantly from 7 DPA to 25 DPA but then started to decrease gradually during 25 DPA to 30 DPA (Fig. 3c). The diversity of proteins increased with seed development, especially from 19 DPA to $25 \mathrm{DPA}$. It is plausible to infer that a large number of genes related to storage protein biosynthesis are expressed during $19 \mathrm{DPA}$ to $25 \mathrm{DPA}$. To test this hypothesis, we identified 16 DEGs related to storage proteins (Additional file 1: Figure S3a) in the transcriptome. The expression of FtPinG0000417000.01 and FtPinG0008579200.01, which are related to glutelin, was upregulated during 19 DPA to 25 DPA (Additional file 1: Figure S3a); these genes were enriched in the molecular function terms nutrient reservoir activity (GO:0045735) and acireductone dioxygenase [iron (II)-requiring] activity (GO:0010309) (Additional file 1: Figure S3a). Glutelin is responsible for some refined baking properties in bread. High-molecular-weight (HMW) and low-molecular-weight (LMW) glutelins are commonly present in these substances. A HMW glutelin of the grass tribe Triticeae can be used as an intraperitoneal disease sensitizer for individuals with the HLA-DQ8 class II antigen receptor gene [62]. Hence, FtPinG0000417000.01 and FtPinG0008579200.01 were good candidate genes related to glutelin biosynthesis during seed development. Storage globulins of 11-12S in the starchy endosperm are also present in some grains [63]. Our data showed that the DEGs encoding the $13 \mathrm{~S}$ globulin seed storage protein and the $11 \mathrm{~S}$ globulin seed storage protein were upregulated mainly from $13 \mathrm{DPA}$ to $19 \mathrm{DPA}$ (Additional file 1: Figure S3a) and belong to the 11S seed storage protein (globulin) family. From a seed development standpoint, starchy endosperm is gradually absorbed by cotyledons during buckwheat seed development (Fig. 1a), providing nitrogen sources for cotyledon development during premetaphase in buckwheat seed development. Those DEGs were enriched in the molecular function term nutrient reservoir activity (GO:0045735) (Additional file 1: Figure S3a). The expression of only FtPinG0004288700.01 was upregulated from 19 DPA to 25 DPA (Additional file 1: Figure S3a). As expected, a large number of genes related to storage protein biosynthesis were expressed from 19
DPA to 25 DPA, and we identified strong candidate genes related to the biosynthesis of these storage proteins.

Tartary buckwheat comprises a large group of widely grown medicinal and edible crops and is considered the main dietary source of rich rutin [15]. In mature buckwheat seeds, rutin accounts for $10 \%$ of the nutrients (Fig. 3e) and is an important secondary metabolite that reduces UV light damage as a key UV-B-absorbing compound [64]. Thus, rutin plays an important role in the development of buckwheat seed. In this study, the content of rutin increased gradually with the development of buckwheat seeds, especially during 25 DPA to 30 DPA (Fig. 3a). Among the genes related to rutin biosynthesis, we identified 5 DEGs from 13 DPA to 25 DPA (Additional file 1: Figure S1a). In addition, the expression patterns of 9 important rutin biosynthesis related genes were verified by RT-qPCR (Fig. 11a). In Fig. 11b, most of these DEGs in the upstream portion of the rutin biosynthesis pathway exhibited higher expression during 13 DPA to 19 DPA. Among them, FtPinG0008236900.01 was matched to the PAL gene. PAL is a key enzyme in plant metabolism and catalyzes the first step of the biosynthesis of phenylpropane skeletons by L-phenylalanine, leading to a variety of natural products. FtPinG0008131000.01 was matched to the CHS gene. The CHS protein is involved in the biosynthesis of flavonoids and plays in a role in the biosynthesis of secondary metabolites. The primary product of this enzyme is 4,2',4',6'-tetrahydroxychalcone, which can then spontaneously isomerize to naringin under certain conditions. FtPinG0002790600.01 was matched to the CHI gene; the encoded protein catalyzes the intramolecular cyclization of bicyclic chalcone to tricyclic (S)-flavanones and is responsible for the isomerization of 4,2',4',6'-tetrahydroxychalcone to naringenin. FtPinG0006907000.01 and FtPinG0006907100.01 were matched to the FLS gene; the FLS protein reacts with dihydroflavonol to produce flavonol, which can react with dihydrokaempferol to produce kaempferol. In turn, kaempferol can lead to the production of quercetin from dihydroquercetin, and catalysis of dihydromyricetin can yield myricetin. The high content of rutin in tartary buckwheat seeds may be related to these DEGs. Overall, we have identified strong candidate genes related to rutin biosynthesis during the development of buckwheat seeds.

\section{Conclusions}

In recent years, the gene regulatory network governing the physiological changes occurring during seed development have receive little attention. The development of tartary buckwheat seeds was characterized by light and electron microscopy. The accumulation of plant hormones and nutrients was measured by high-performance liquid chromatography, and the expression of key genes was analyzed with the support of the genome of tartary 

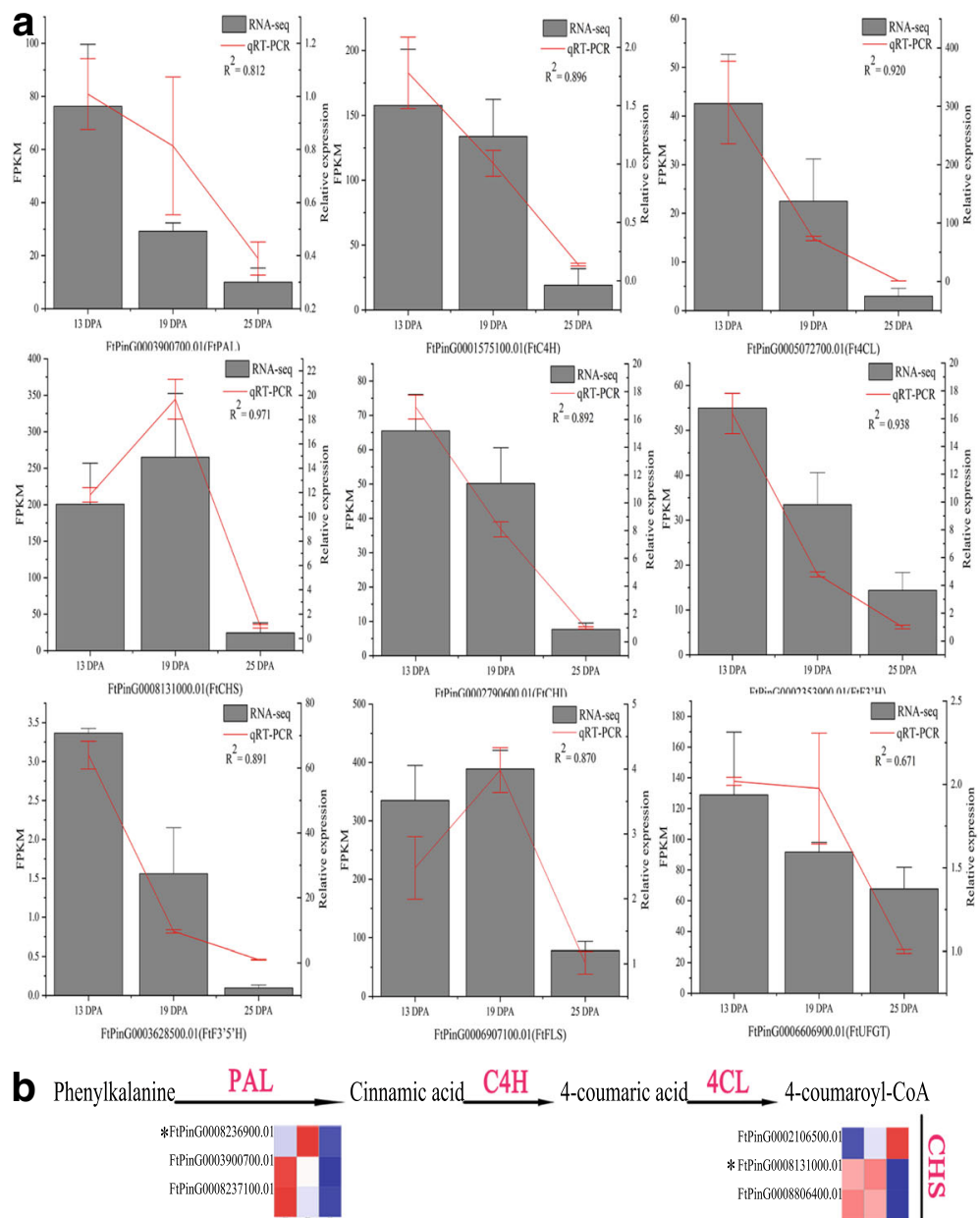

Cinnamic acid $\stackrel{\mathrm{CH}}{\longrightarrow}$ 4-coumaric acid $\stackrel{\text { 4CL }}{\longrightarrow}$ 4-coumaroyl-CoA

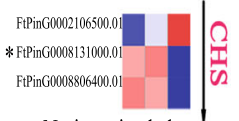

Naringenin chalcone

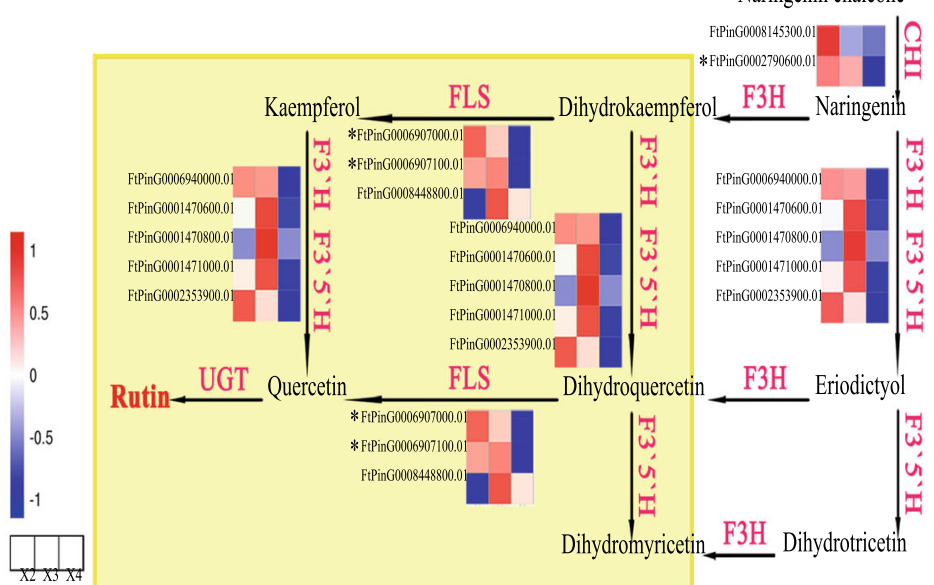

Fig. 11 DEGs involved in rutin biosynthesis pathway. a RT- qPCR confirmation of 9 rutin biosynthesis related genes. b A simplified representation of the flavonoid biosynthetic pathway (adopted from the KEGG PATHWAY Database: http://www.genome.jp/kegg/pathway.html) shows the following enzymes: phenylalanine ammonia-lyase (PAL), cinnamate-4-hydroxylase (C4H), 4-coumarate Co A ligase (4CL), chalcone synthase (CHS), chalcone isomerase $(\mathrm{CHI})$, flavanone-3'-hydroxylase $\left(\mathrm{F} 3^{\prime} \mathrm{H}\right)$, flavanone-3-hydroxylase $(\mathrm{F} 3 \mathrm{H})$, flavonol synthase (FLS), glucosyl/rhamnosyl transferase, and flavanone-3'-5'-hydroxylase $\left(F 3^{\prime} 5^{\prime} H\right)$. The expression value of each gene is colored in $\log _{10}(\mathrm{FPKM})$ in three samples X2 (13 DPA), X3 (19 DPA), and X4 (25 DPA). * indicate the gene was differentially expressed during 13 DPA to 25DPA 
buckwheat. By analyzing the relationship between plant hormone changes and seed development, we studied the embryo cell expansion and primary dormancy of tartary buckwheat seeds. In addition, by analyzing the relationship between nutritional changes and seed development, we determined the gene regulatory network controlling the accumulation of rutin, starch, storage protein and soluble sugars. We have provided abundant genomic resources for tartary buckwheat and Polygonaceae communities and carried out novel molecular studies on the correlations between the physiological changes in and the seed development of tartary buckwheat.

\section{Methods}

\section{Plant materials and phenotype investigations}

Seeds of tartary buckwheat (Xiqiao No. 2) were collected in 2016 from the experimental field of the College of Life Science, Sichuan Agricultural University (Lat. 29 $97^{\prime}$ $\mathrm{N}, 102^{\circ} 97^{\prime}$ E, Alt. $580 \mathrm{~m}$ ), China. We observed the development of seeds from anthesis until maturation in April-May, 2016. Seeds were collected manually every two days from the onset of seed set until maturity (Fig. 1), covering a total span of 30 days, after which the size and weight of the developing seeds were measured. For phytohormone, rutin and nutrient sampling, seeds at five development stages $(7,13,19,25$, and $30 \mathrm{DPA})$ were collected from the same individual. For the transcriptome sampling, seeds at three development stages (13, 19, and 25 DPA) were collected from the same individual. In addition, seeds at the same development stage were collected from three replicate plants. The samples were immediately flash frozen in liquid nitrogen and stored at $-80^{\circ} \mathrm{C}$ for further use.

\section{Measurement of phytohormone contents}

Fresh samples that were weighed to approximately $0.5 \mathrm{~g}$ were ground in liquid nitrogen. The powder was subsequently homogenized in $10 \mathrm{~mL}$ of $80 \%$ methanol, after which the solution was stirred overnight at $4^{\circ} \mathrm{C}$. This suspension was then centrifuged at $12000 \mathrm{rpm}$ for 10 min under refrigeration $\left(4^{\circ} \mathrm{C}\right)$. The supernatant was collected, after which $5 \mathrm{~mL}$ of $80 \%$ methanol was added to the residue. Similarly, the supernatant was collected after centrifugation. The pooled supernatant $(\sim 15 \mathrm{~mL})$ was flash evaporated at $36^{\circ} \mathrm{C}$ until the methanol vaporized $(\sim 3 \mathrm{~mL})$. A bottle was washed with $5 \mathrm{~mL}$ of ultrapure water, and then the water was combined with the residual liquid $(\sim 3 \mathrm{~mL})$. The solution was decolorized with $15 \mathrm{~mL}$ of diethyl ether three times, after which the ether phase was discarded. The aqueous phase was collected and basified to $\mathrm{pH} 8.0$ with $0.1 \mathrm{M} \mathrm{Na}_{2} \mathrm{HPO}_{3}$. The basified extract was kept in a shaker for 30 min with 50 $\mathrm{mg}$ of polyvinylpyrrolidone (PVP) at $4^{\circ} \mathrm{C}$. In addition, the extract was centrifuged at $12000 \mathrm{rpm}$ for $10 \mathrm{~min}$. The supernatant was subsequently collected and was acidified to $\mathrm{pH} 3.0$ with $0.2 \mathrm{M}$ citric acid. The resultant solution was partitioned three times against $5 \mathrm{~mL}$ of ethylacetate, after which the aqueous phase was discarded. The pooled ethylacetate phase $(\sim 15 \mathrm{~mL})$ was flash evaporated at $36^{\circ} \mathrm{C}$ to near dryness. Afterward, the residue was dissolved in $1 \mathrm{~mL}$ of methanol [65-67].

The sample was filtered through a nylon 66 filter $(25$ $\mathrm{mm}$ diameter, $0.45 \mu \mathrm{m}$ pore size) prior to injection into a high-performance liquid chromatograph. HPLC analysis was performed on an Agilent 1260 system using a C18-ODS $(3.5 \mu \mathrm{m} \times 150 \mathrm{~mm} \times 4.6 \mathrm{~mm})$ column (Agilent, USA) and a UV/VIS detector. An injection volume of 10 $\mu \mathrm{L}$, column temperature of $35^{\circ} \mathrm{C}$, flow rate of $1 \mathrm{~mL} \mathrm{~min}^{-1}$, and run time of $10 \mathrm{~min}$ were maintained for all analyses. The system was calibrated with external standards of IAA, $\mathrm{GA}_{3}, \mathrm{ABA}$, and ZT. For detection, separation was performed with a mixture of methanol and distilled water containing $0.6 \%$ acetic acid $(\mathrm{V}: \mathrm{V}=50: 50)$ following isocratic elution. The elutant was scanned at $257 \mathrm{~nm}$.

\section{Measurement of rutin contents}

The seeds of tartary buckwheat were dried at $60^{\circ} \mathrm{C}$ for $4 \mathrm{~h}$, milled into a fine powder, and filtered through a 0.177 $\mathrm{mm}$ sieve. A total of $0.10 \mathrm{~g}$ of dry powder for each tissue was mixed with $1 \mathrm{~mL}$ of methanol and incubated at $60^{\circ} \mathrm{C}$ using an ultrasonic bath for $2 \mathrm{~h}$. The mixture was then centrifuged at $4000 \mathrm{r} \mathrm{min}^{-1}$ for $10 \mathrm{~min}$, after which the supernatant was collected. The supernatant's $\mathrm{pH}$ value was adjusted to 6 using $0.1 \mathrm{~mol} \mathrm{~L}^{-1}$ phosphoric acid and then filtered through $0.22 \mu \mathrm{m}$ membrane filter. High-performance liquid chromatography was performed on an Agilent 1260 system using an Agilent C18 column (4.6 $\mathrm{mm} \times 150 \mathrm{~mm}, 5 \mu \mathrm{m})$ (Agilent Technologies, USA) at $30^{\circ} \mathrm{C}$. The mobile phase consisted of methanol:0.5\% phosphoric acid $(\mathrm{V} / \mathrm{V})=40: 60$ at flow rate of $1 \mathrm{~mL} \mathrm{~min}^{-1}$ with a total injection volume of $10 \mu \mathrm{L}$. The rutin quantity was estimated based on the linear calibration curve of standard rutin hydrate (98\%) (Yuanye, China) at a detection wavelength of $257 \mathrm{~nm}$. Three independent sample analyses were performed for each tissue [68].

\section{Measurement of nutrient contents}

The contents of total soluble sugars in buckwheat seeds at different developmental periods were measured by anthrone- $\mathrm{H}_{2} \mathrm{SO}_{4}$ colorimetry. Ground sample tissue $(0.07 \mathrm{~g})$ and $7 \mathrm{~mL}$ of distilled water were put in a $10 \mathrm{~mL}$ graduated test tube that was closed with a stopper. The mixture was then extracted in a water bath for $30 \mathrm{~min}$ at $100^{\circ} \mathrm{C}$ and centrifuged at $4000 \times \mathrm{g}$ for $5 \mathrm{~min}$, after which the supernatant was transferred to a $50 \mathrm{~mL}$ volumetric flask (extracted twice). Afterward, $100 \mu \mathrm{L}$ of the extract was pipetted into the test tube, and $3 \mathrm{ml}$ of anthrone reagent was added, after which the tube was placed in a boiling water bath for $10 \mathrm{~min}$. The change in 
absorbance at $620 \mathrm{~nm}$ was then measured, after which the soluble sugar content was calculated according to the standard curve [69].

The total protein content was measured using the Coomassie brilliant blue G-250 method. A standard protein solution and a Coomassie brilliant blue G-250 solution were prepared, and fresh seeds $(0.5 \mathrm{~g})$ were ground in $10 \mathrm{~mL}$ of distilled water using a mortar and pestle. After the solution was centrifuged at $4000 \times \mathrm{g}$ for 10 min, the supernatant was transferred to a new tube to assay the total protein content, and the change in absorbance at $595 \mathrm{~nm}$ was measured [69].

The contents of starch in the seeds of buckwheat were measured by anthrone- $\mathrm{H}_{2} \mathrm{SO}_{4}$ colorimetry [70]. The solid residues that extract soluble sugars were used to test the starch content. First, $20 \mathrm{~mL}$ of hot water was added to the tube that contained solid residues. An extraction in a water bath $\left(100^{\circ} \mathrm{C}\right)$ for $15 \mathrm{~min}$ then occurred, after which $2 \mathrm{~mL}$ of $9.2 \mathrm{~mol} \mathrm{~L}^{-1}$ perchloric acid was added; the water bath $\left(100^{\circ} \mathrm{C}\right)$ extraction then continued for $15 \mathrm{~min}$. After cooling and mixing the extract, the filtrate was brought to a constant volume in a $50 \mathrm{~mL}$ measuring flask. Afterward, $100 \mu \mathrm{L}$ of the extract was pipetted into the test tube, and $3 \mathrm{~mL}$ of anthrone reagent was added. The extract was then placed in a boiling water bath for $10 \mathrm{~min}$. The change in absorbance at $620 \mathrm{~nm}$ was then measured, and the starch content was calculated according to the standard curve [69].

\section{Statistical analysis}

All the data were analyzed by analysis of variance using the Origin Pro 2017 statistics program, and the means were compared by the least significant difference test (LSD) at the 0.05 level of significance. The Pearson's correlation coefficient between DPA, rutin, starch, total proteins, soluble sugars, $\mathrm{ZT}, \mathrm{GA}_{3}$, IAA, $\mathrm{ABA}$, transverse diameter, longitudinal diameter, transverse diameter $\times$ longitudinal diameter, and seed weight were analyzed by Origin Pro 2017 software.

\section{Total RNA extraction, cDNA Library construction, and high-throughput sequencing}

The total RNA samples were extracted using the TRIzol reagent (Invitrogen) and were then treated by RNase-free DNase I (Takara) to remove the genomic DNA. mRNA libraries were constructed according to the standard protocols provided by Illumina. The quality of mRNA, including purity, quantity and integrity, was tested using Nanodrop, Qubit, and Agilent 2100 systems. The mRNA was enriched using Dynabeads oligos (dTs) (Dynal; Invitrogen) and fragmented using fragmentation buffer. Double-stranded cDNAs were synthesized using both reverse transcriptase (Superscript II; Invitrogen) and random hexamer primers and were further purified using
AMPure XP beads. Finally, the purified double-stranded cDNA samples were further enriched by PCR to construct the final cDNA libraries that were sequenced using a HiSeq 4000 (150 bp paired ends) by Novogene (China). All raw sequence read data were uploaded to the NCBI Sequence Read Archive (SRA, http://www.ncbi.nlm.nih.gov/Traces/sra) under accession number GSE111937.

\section{Map sequencing results and differential expression analysis}

Adaptor sequences and low-quality sequences were removed from the raw reads $(Q<20)$. The clean reads were aligned to the reference genome sequences of the tartary buckwheat (Pinku1) genome (http://www.mbkbase.org/ Pinku1/) using TopHat (v2.0.12). The default parameters were used, allowing mismatches of no more than two bases. A reference-based assembly of all the reads was performed using the Cufflinks v2.1.1 reference annotation-based transcript (RABT) assembly method. The assembled transcript fragments were compared with the reference annotation to predict new genes and novel exons and to optimize gene structures using Cuffcompare.

Gene expression differences in the different sample pairs were detected using the DESeq package (v1.10.1). In this study, to investigate the genes involved in tartary buckwheat seed development, the two sample pairs (13 DPA vs 19 DPA and 19 DPA vs 25 DPA) were used to investigate the differentially expressed genes under different conditions. The P-value threshold was determined using the FDR for multiple tests [71]. The thresholds were set using an FDR $\leq 0.05$ and the absolute value of $\log _{2}$ (fold change) with FPKM $\geq 1$ to determine significant differences in gene expression. The FPKM was used to eliminate the influence of different gene lengths and sequencing discrepancies on the quantification of gene expression to ensure direct comparison of gene expression between different sample pairs [72].

Functional classification of differentially expressed genes An analysis of functional enrichment, including GO, was performed to identify which DEGs were significantly enriched in GO terms. GO enrichment of the DEGs was conducted using the GOseq $\mathrm{R}$ package (release 2.12). GO terms with a corrected P-value $<0.05$ were considered significantly enriched by the differentially expressed genes. The GO annotations were functionally classified using WEGO software for gene function distributions. KOBAS software (v2.0) was used to identify the statistical enrichment of the differentially expressed genes in the KEGG pathways. The pathways with an FDR value of less than 0.05 were considered those genes showing significantly differential expression. 


\section{Real-Time PCR confirmation of differentially expressed genes}

Quantitative real-time PCR analysis was performed to confirm transcriptome results. The corresponding sequences of these genes were obtained from the tartary buckwheat (Pinku1) genome sequence database. The RT-qPCR primers were designed according to the transcript sequences of 20 genes using Primer3 software (http://frodo.wi.mit.edu/) (Additional file 1: Table S7). The FtH3 gene was used as the internal control. RT-qPCR experiments were replicated at least three times.

First-strand cDNA was synthesized from $1 \mathrm{mg}$ of DNase I-treated RNA samples in a $40 \mu \mathrm{L}$ reaction solution with random primers, using a PrimeScript RT Reagent Kit with gDNA Eraser (TaKaRa). Standard RTqPCR was performed using SYBR Premix Ex Taq II (TaKaRa) on a CFX96 Real Time System (BioRad). Data were analyzed by the $2^{-(\Delta \Delta \mathrm{Ct})}$ method to obtain relative mRNA expression data [73].

\section{Additional file}

Additional file 1: Figure S1. DEGs related to flavonoid in three samples. Figure S2. DEGs related to starch in three samples. Figure S3. DEGs related to storage protein in three samples. Table S1. Quality of the RNA sequencing data. Table S2. Information of reads aligned to the reference genome sequence. Table S3. Pearson correlation between RNA-seq data from different samples. Table S4. List of genes were related to phytohormones during seed development. Table S5. List of genes were related to flavonoid during seed development. Table S6. Validation of the transcriptome data by qRT-PCR. Table S7. Primers sequences. (PDF $817 \mathrm{~kb}$ )

\begin{abstract}
Abbreviations
ABA: Abscisic acid; ABRE: ABA-responsive element; AGPase: ADP-glucose pyrophosphorylase; ARFs: Auxin response factors; AuxREs: Auxin-responsive promoter elements; BE: Branching enzyme; $\mathrm{CHI}$ : Chalcone isomerase; CHS: Chalcone synthase; DBE: Starch debranching enzyme;

DEGs: Differentially expressed genes; DPA: Days postanthesis; DW: Dry weight; FDR: False discovery rate; FLS: Flavonol synthase; FPKM: Fragments per kilobase of transcripts per million mapped fragments; FW: Fresh weight; $\mathrm{GA}_{3}$ : Gibberellin; GBSS: Granule-bound starch synthase; GO: Gene Ontology; HMW: High-molecular-weight; HPLC: High efficiency liquid chromatography; IAA: Indole-3-acetic acid; LMW: Low-molecular-weight; LSD: Least significant difference test; PAL: Phenylalanine ammonia-lyase; PCA: Principal component analysis; PP2Cs: 2C phosphatases; PVP: Polyvinylpyrrolidone; PYL10: Abscisic acid receptor; PYL4: Abscisic acid receptor; RNA-seq: RNA sequencing; RTqPCR: Real-time Quantitative polymerase chain reaction; SS: Soluble starch synthase; SUS: Sucrose synthase; UGPase: UDP-glucose pyrophosphorylase; ZT: Zeatin
\end{abstract}

\section{Acknowledgments}

We are very grateful to the editor and reviewers for critically evaluating the manuscript and providing constructive comments for its improvement.

\section{Funding}

This research was supported by the National Natural Science Foundation of China (31500289).

\section{Availability of data and materials}

The datasets supporting the results presented in this manuscript are included within the article (and its additional files). All raw sequence read data were uploaded to the NCBI Sequence Read Archive (SRA, http:// www.ncbi.nlm.nih.gov/Traces/sra) under accession number GSE111937.

\section{Authors' contributions}

ML planned and designed the research, analyzed data and wrote the manuscript. ZM used RT-qPCR to validate the digital expression from RNAseq and made analysis and interpretation of these data. ZM and TZ measured phytohormones. WS, YZ and WJ measured nutrients. JZ, YC and YT planted and collected plant materials. QW, ZT, TB and CL contributed the reagents and analytical tools. HC supervised the research. ML and ZM contributed equally. All of the authors read and approved the final manuscript.

\section{Ethics approval and consent to participate}

This study did not directly involve humans or animals.

Seeds of tartary buckwheat (Xiqiao No. 2) were collected in 2016 from the experimental field of the College of Life Science, Sichuan Agricultural University (Lat. $29^{\circ} 97^{\prime} \mathrm{N}, 102^{\circ} 97^{\prime} \mathrm{E}$, Alt. 580 m), China. Collection of plant materials complied with the institutional, national and international guidelines. The research conducted complied with all institutional and national guidelines.

\section{Consent for publication}

Not applicable.

\section{Competing interests}

The authors declare that they have no competing interests.

\section{Publisher's Note}

Springer Nature remains neutral with regard to jurisdictional claims in published maps and institutional affiliations.

Received: 19 May 2018 Accepted: 24 August 2018

Published online: 31 August 2018

\section{References}

1. Ohsako T, Yamane K, Ohnishi O. Two new Fagopyrum (Polygonaceae) species, F. gracilipedoides and F. jinshaense from Yunnan, China. Japanese J Genet. 2002;77(6):399-408.

2. Bankevich A, Nurk S, Antipov D, Gurevich AA, Dvorkin M, Kulikov AS, Lesin VM, Nikolenko SI, Pham S, Prjibelski AD. SPAdes: a new genome assembly algorithm and its applications to single-cell sequencing. J Comput Biol. 2012;19(5):455-77.

3. Li A, S-p H. Flora of China. Volume 4: Fagopyrum tataricum. Flora China. 2004;5(1):322.

4. Lee CC, Shen SR, Lai YJ, Wu SC. Rutin and quercetin, bioactive compounds from tartary buckwheat, prevent liver inflammatory injury. Food Funct. 2013; 4(5):794-802.

5. Matilla AJ, MatillaváZquez MA. Involvement of ethylene in seed physiology. Plant Sci. 2008;175(1-2):87-97.

6. Kermode AR, Finchsavage BE, Black M, Pritchard HW. Desiccation sensitivity in orthodox and recalcitrant seeds in relation to development. In: Desiccation \& Survival in Plants Drying Without Dying; 2002.

7. Chen J, Zeng B, Zhang M, Xie S, Wang G, Hauck A, Lai J. Dynamic transcriptome landscape of maize embryo and endosperm development. Plant Physiol. 2014;166(1):252-64.

8. Kreft S, Knapp M, Kreft I. Extraction of rutin from buckwheat (Fagopyrum esculentumMoench) seeds and determination by capillary electrophoresis. J Agric Food Chem. 1999;47(11):4649-52.

9. Bonafaccia G, Gambelli L, Fabjan N, Kreft I. Trace elements in flour and bran from common and tartary buckwheat. Food Chemistry. 2003;83(1):1-5.

10. Eggum DBO, Kreft I, Javornik B. Chemical composition and protein quality of buckwheat (Fagopyrum esculentum Moench). Plant Foods Hum Nutr. 1980;30(3-4):175-9.

11. Skrabanja V, Kreft I, Golob T, Modic M, Ikeda S, Ikeda K, Kreft S, Bonafaccia G, Knapp M, Kosmelj K. Nutrient content in buckwheat milling fractions. Cereal Chem. 2004:81(2):172-6.

12. Hollender CA, Geretz AC, Slovin JP, Liu Z. Flower and early fruit development in a diploid strawberry, Fragaria vesca. Planta. 2012; 235(6):1123-39

13. Trapnell C, Williams BA, Pertea G, Mortazavi A, Kwan G, van Baren MJ, Salzberg SL, Wold BJ, Pachter L. Transcript assembly and quantification by 
RNA-Seq reveals unannotated transcripts and isoform switching during cell differentiation. Nat Biotechnol. 2010;28(5):511-5.

14. Srivastava LM. Plant growth and development: hormones and environment. Boston: Academic Press; 2002.

15. Zielińska D, Turemko M, Kwiatkowski J, Zieliński H. Evaluation of flavonoid contents and antioxidant capacity of the aerial parts of common and tartary buckwheat plants. Molecules. 2012;17(8):9668-82.

16. Vida Skrabanja, §, Ivan Kreft A, Björck IM: Nutritional Properties of Starch in Buckwheat Products: Studies in Vitro and in Vivo. J Agric Food Chem 2001, 49(1):490.

17. Osborne TB. The vegetable proteins: Longmans, Green and Co; 1924. https://www.biodiversitylibrary.org/item/58602\#page/5/mode/1up.

18. Chapman EJ, Estelle M. Mechanism of auxin-regulated gene expression in plants. Annu Rev Genet. 2009;43(1):265.

19. Perrotrechenmann C. Cellular Responses to Auxin: Division versus Expansion. Cold Spring Harb Perspect Biol. 2010;2(5):a001446.

20. Liscum E, Reed JW. Genetics of Aux/IAA and ARF action in plant growth and development. Netherlands: Springer; 2002.

21. Vidaurre DP, Ploense S, Krogan NT, Berleth T. AMP1 and MP antagonistically regulate embryo and meristem development in Arabidopsis. Development. 2007;134(14):2561-7.

22. Hardtke CS, Ckurshumova W, Vidaurre DP, Singh SA, Stamatiou G, Tiwari SB, Hagen G, Guilfoyle TJ, Berleth T. Overlapping and non-redundant functions of the Arabidopsis auxinresponse factors MONOPTEROS and NONPHOTOTROPIC HYPOCOTYL4. Development. 2004;131(5):1089-100.

23. Hagen G, Guilfoyle T. Auxin-responsive gene expression: genes, promoters and regulatory factors. Plant Mol Biol. 2002:49(3-4):373-85.

24. Park JE, Kim YS, Yoon HK, Park CM. Functional characterization of a small auxin-up RNA gene in apical hook development in Arabidopsis. Plant Sci. 2007;172(1):150-7.

25. Finchsavage WE, Clay HA, Blake PS, Browning G. Seed Development in the Recalcitrant Species Quercus robur L: Water Status and Endogenous Abscisic Acid Levels. J Exp Bot. 1992;43(250):671-9.

26. Kong $L$, Attree $S M$, Fowke LC. Changes of endogenous hormone levels in developing seeds, zygotic embryos and megagametophytes in Picea glauca. Physiol Plant. 1997;101(1):23-30.

27. Karssen CM, DI BDS, Breekland AE, Koornneef M. Induction of dormancy during seed development by endogenous abscisic acid: studies on abscisic acid deficient genotypes of Arabidopsis thaliana (L.) Heynh. Planta. 1983; 157(2):158-65.

28. Arnold RLB, Fenner M, Edwards PJ. Changes in germinability, ABA content and $A B A$ embryonic sensitivity in developing seeds of Sorghum bicolor (L.) Moench. induced by water stress during grain filling. New Phytol. 1991; 118(2):339-47.

29. Morandi EN, Schussler JR, Brenner ML. Photoperiodically Induced Changes in Seed Growth Rate of Soybean as Related to Endogenous Concentrations of ABA and Sucrose in Seed Tissues. Ann Bot. 1990;66(5):605-11.

30. Bo H, Wan XR, Liu XH, Guo DL, Ling L. Abscisic acid (aba)-mediated inhibition of seed germination involves a positive feedback regulation of aba biosynthesis in Arachis hypogaea L. Afr J Biotechnol. 2010;9(11):1578-86.

31. Bangerth F. Endogenous hormone levels in explants and in embryogenic and non-embryogenic cultures of carrot. Physiol Plant. 2001:111(3):389-95.

32. Crouch ML, Sussex IM. Development and storage-protein synthesis in Brassica napus L. embryos in vivo and in vitro. Planta. 1981;153(1):64-74.

33. Delisle AJ, Crouch ML. Seed Storage Protein Transcription and mRNA Levels in Brassica napus during Development and in Response to Exogenous Abscisic Acid. Plant Physiol. 1989;91 (2):617-23.

34. Spencer TM, Kitto SL. Measurement of endogenous ABA levels in chilled somatic embryos of carrot by immunoassay. Plant Cell Rep. 1988;7(5):352-5.

35. Hwm H. A critical update on seed dormancy. I. Primary dormancy. Seed Science Research. 1995;5(2):61-73.

36. Leubner-Metzger G. Functions and regulation of $\beta-1,3-g$ lucanases during seed germination, dormancy release and after-ripening. Seed Sci Res. 2003; 13(1):17-34

37. Nambara E, Marion-Poll A. ABA action and interactions in seeds. Trends Plant Sci. 2003;8(5):213.

38. Bewley JD. Seed Germination and Dormancy. Plant Cell. 1997;9(7):1055-66.

39. Singh DP, Jermakow AM, Swain SM. Gibberellins are required for seed development and pollen tube growth in Arabidopsis. Plant Cell. 2002; 14(12):3133.
40. Koornneef M, Bentsink L, Hilhorst H. Seed dormancy and germination. Curr Opin Plant Biol. 2002;5(1):33.

41. Finkelstein R, Reeves W, Ariizumi T, Steber C. Molecular aspects of seed dormancy. Annu Rev Plant Biol. 2008;59(59):387.

42. Finchsavage WE, Leubnermetzger G. Seed dormancy and the control of germination. New Phytol. 2006;171(3):501-23.

43. Rodriguez L, Gonzalezguzman M, Diaz M, Rodrigues A, Izquierdogarcia AC, Peiratsllobet M, Fernandez MA, Antoni R, Fernandez D, Marquez JA. C2domain abscisic acid-related proteins mediate the interaction of PYR/PYL/ RCAR abscisic acid receptors with the plasma membrane and regulate abscisic acid sensitivity in Arabidopsis. Plant Cell. 2014;26(12):4802-20.

44. Zhang X, Jiang L, Wang G, Yu L, Zhang Q, Xin Q, Wu W, Gong Z, Chen Z. Structural Insights into the Abscisic Acid Stereospecificity by the ABA Receptors PYR/PYL/RCAR. Plos One. 2013;8(8):e67477.

45. Hao Q, Yin P, Li W, Wang L, Yan C, Lin Z, Wu JZ, Wang J, Yan SF, Yan N. The molecular basis of ABA-independent inhibition of PP2Cs by a subclass of PYL proteins. Mol Cell. 2011;42(5):662-72.

46. Santiago J, Rodrigues A, Saez A, Rubio S, Antoni R, Dupeux F, Park SY, Márquez JA, Cutler SR, Rodriguez PL. Modulation of drought resistance by the abscisic acid receptor PYL5 through inhibition of clade A PP2Cs. Plant J Cell Mol Biol. 2009;60(4):575.

47. Lopezmolina L, Mongrand S, Chua NH. A postgermination developmental arrest checkpoint is mediated by abscisic acid and requires the ABI5 transcription factor in Arabidopsis. Proc Natl Acad Sci U S A. 2001;98(8):4782-7.

48. Bensmihen S, Rippa S, Lambert G, Jublot D, Pautot V, Granier F, Giraudat J, Parcy F. The homologous ABI5 and EEL transcription factors function antagonistically to fine-tune gene expression during late embryogenesis. Plant Cell. 2002;14(6):1391.

49. Carles C, Bies-Etheve N, Aspart L, Léon-Kloosterziel KM, Koornneef M, Echeverria M, Delseny M. Regulation of Arabidopsis thaliana Em genes: role of ABI5. Plant J Cell Mol Biol. 2002;30(3):373-83.

50. Lopez-Molina L, Mongrand S, Mclachlin DT, Chait BT, Chua NH. ABI5 acts downstream of $\mathrm{ABI} 3$ to execute an $\mathrm{ABA}$-dependent growth arrest during germination. Plant J. 2002;32(3):317-28.

51. Brocard IM, Lynch TJ, Finkelstein RR. Regulation and Role of the Arabidopsis Abscisic Acid-Insensitive 5 Gene in Abscisic Acid, Sugar, and Stress Response. Plant Physiol. 2002;129(4):1533-43.

52. Lu C, Han MH, Guevara-Garcia A, Fedoroff NV. Mitogen-activated protein kinase signaling in postgermination arrest of development by abscisic acid. Proc Natl Acad Sci U S A. 2002:99(24):15812.

53. Nakashima K, Fujita Y, Katsura K, Maruyama K, Narusaka Y, Seki M, Shinozaki K, Yamaguchi-Shinozaki K. Transcriptional Regulation of ABI3- and ABAresponsive Genes Including RD29B and RD29A in Seeds, Germinating Embryos, and Seedlings of Arabidopsis. Plant Mol Biol. 2006;60(1):51-68.

54. Fujita Y, Fujita M, Satoh R, Maruyama K, Parvez MM, Seki M, Hiratsu K, Ohmetakagi M, Shinozaki K, Yamaguchishinozaki K. AREB1 is a transcription activator of novel ABRE-dependent ABA signaling that enhances drought stress tolerance in Arabidopsis. Plant Cell. 2005;17(12):3470-88.

55. Kim S, Kang JY, Cho DI, Park JH, Kim SY. ABF2, an ABRE-binding bZIP factor, is an essential component of glucose signaling and its overexpression affects multiple stress tolerance. Plant Journal. 2004;40(1):75-87.

56. Uno Y, Furihata T, Abe H, Yoshida R, Shinozaki K, Yamaguchishinozaki K. Arabidopsis basic leucine zipper transcription factors involved in an abscisic acid-dependent signal transduction pathway under drought and highsalinity conditions. Proc Natl Acad Sci U S A. 2000:97(21):11632-7.

57. Gãmez-Arjona FM, Li J, Raynaud S, Baroja-Fernández E, Muñoz FJ, Ovecka M, Ragel P, Bahaji A, Pozueta-Romero J, Mérida Á. Enhancing the expression of starch synthase class IV results in increased levels of both transitory and long-term storage starch. Plant Biotechnol J. 2011;9(9):1049-60.

58. Szydlowski N, Ragel P, Raynaud S, Lucas MM, Roldán I, Montero M, Muñoz FJ, Ovecka M, Bahaji A, Planchot V. Starch granule initiation in Arabidopsis requires the presence of either class IV or class III starch synthases. Plant Cell. 2009;21(8):2443-57.

59. Roldán I, Wattebled F, Mercedes ML, Delvallé $D$, Planchot $V$, Jiménez $S$, Pérez R, Ball S, D'Hulst C, Mérida A. The phenotype of soluble starch synthase IV defective mutants of Arabidopsis thaliana suggests a novel function of elongation enzymes in the control of starch granule formation. Plant J. 2007:49(3):492-504.

60. Kazłowski B, Chen M, Chao P, Lai C, Ko Y. Identification and Roles of Proteins for Seed Development in Mungbean (Vigna radiata L.) Seed Proteomes. J Agric Food Chem. 2013;61 (27):6650-9. 
61. Hajduch M, Ganapathy A, Stein JW, Thelen JJ. A systematic proteomic study of seed filling in soybean. Establishment of high-resolution two-dimensional reference maps, expression profiles, and an interactive proteome database. Plant Physiol. 2005:137(4):1397-419.

62. Dewar DH, Amato M, Ellis HJ, Pollock EL, Gonzalezcinca N, Wieser H, Ciclitira PJ. The toxicity of high molecular weight glutenin subunits of wheat to patients with coeliac disease. Eur J Gastroenterol Hepatol. 2006;18(5):483.

63. Casey R. Distribution and Some Properties of Seed Globulins. In: Shewry P, Casey R, editors. Seed proteins. Dordrecht: Springer Netherlands; 1999. p. 159-69.

64. Bieza K, Lois R. An Arabidopsis Mutant Tolerant to Lethal Ultraviolet-B Levels Shows Constitutively Elevated Accumulation of Flavonoids and Other Phenolics. Plant Physiol. 2001;126(3):1105-15.

65. Xu X. The Role of Gibberellin, Abscisic Acid, and Sucrose in the Regulation of Potato Tuber Formation In vitro. Plant Physiol. 1998;117(2):575-84.

66. Baydar H. Correlations Between Changes in the Amount of Endogenous Phytohormones and Flowering in the Safflower (Carthamus tinctorius L.). Turkish J Biol. 2014;4:421-6

67. Mwange KN, Hou HW, Cui KM. Relationship between endogenous indole-3acetic acid and abscisic acid changes and bark recovery in Eucommia ulmoides Oliv. after girdling. J Exp Bot. 2003;54(389):1899-907.

68. Zhang L, Li X, Ma B, Gao Q, Du H, Han Y, Li Y, Cao Y, Qi M, Zhu Y. The Tartary Buckwheat Genome Provides Insights into Rutin Biosynthesis and Abiotic Stress Tolerance. Mol Plant. 2017;10(9):1224

69. Yin Y, Dong X. Seed biology. In: Li G, editor. Seed Experiment Technology. Beijing: China Agriculture Press; 2008. p. 21-5.

70. Ye B, Zhu X. Extraction and separation technology of biological macromolecules. In: Tian J, editor. Basic Biological Science Experiment: Plant-Based. Beijing: Higher Education Press; 2007. p. 424-9.

71. Benjamini Y, Hochberg Y. Controlling the False Discovery Rate: A Practical and Powerful Approach to Multiple Testing. J Royal Stat Soc. 1995;57(1):289-300.

72. Mortazavi A, Williams BA, Mccue K, Schaeffer L, Wold B. Mapping and quantifying mammalian transcriptomes by RNA-Seq. Nat Methods. 2008; 5(7):621

73. Kenneth STL. Analysis of Relative Gene Expression Data Using RT-qPCR pdf. Methods. 2001;25:402-8.

Ready to submit your research? Choose BMC and benefit from:

- fast, convenient online submission

- thorough peer review by experienced researchers in your field

- rapid publication on acceptance

- support for research data, including large and complex data types

- gold Open Access which fosters wider collaboration and increased citations

- maximum visibility for your research: over $100 \mathrm{M}$ website views per year

At $\mathrm{BMC}$, research is always in progress.

Learn more biomedcentral.com/submissions 